\title{
molecules
}

ISSN 1420-3049

www.mdpi.com/journal/molecules

Article

\section{Synthesis and Cytotoxic Activity of a New Group of Heterocyclic Analogues of the Combretastatins}

\author{
Alla V. Lipeeva ${ }^{1}$, Elvira E. Shults ${ }^{1, *}$, Makhmut M. Shakirov ${ }^{1}$, Mikhail A. Pokrovsky ${ }^{2}$ and \\ Andrey G. Pokrovsky ${ }^{2}$ \\ 1 Laboratory of Medicinal Chemistry, Novosibirsk Institute of Organic Chemistry, Siberian Branch, \\ Russian Academy of Sciences, Lavrentjev Avenue 9, Novosibirsk 630090, Russia; \\ E-Mails: mond_05@list.ru (A.V.L.); mmsh@nioch.nsc.ru (M.M.S.) \\ 2 Medicinal Department, Novosibirsk State University, Pirogova St. 2, Novosibirsk 630090, Russia; \\ E-Mails: miha.pokrovsky@gmail.com (M.A.P.); decan@medf.nsu.ru (A.G.P.) \\ * Author to whom correspondence should be addressed; E-Mail: schultz@nioch.nsc.ru; \\ Tel.: +7-383-330-8533; Fax: +7-383-330-4752.
}

Received: 15 April 2014; in revised form: 3 June 2014 / Accepted: 5 June 2014 /

Published: 11 June 2014

\begin{abstract}
A series of new analogs of combretastatin A-4 $(\mathrm{CA}-4,1)$ with the A or B-ring replaced by a 3-oxo-2,3-dihydrofurocoumarin or a furocoumarin residue have been designed and synthesized by employing a cross-coupling approach. All the compounds were evaluated for their cytotoxic activity with respect to model cancer cell lines (CEM-13, MT-4, U-937) using conventional MTT assays. Structure-activity relationship analysis reveals that compounds $\mathbf{2}, \mathbf{3}, \mathbf{6}-\mathbf{8}$ in which the $(Z)$-styryl substituent was connected to the 2-position of the 3-oxo-2,3-dihydrofurocoumarin core, demonstrated increased potency compared to 3-(Z)-styrylfurocoumarins 4, 5, 9-11. The methoxy-, hydroxyl- and formyl- substitution on the aromatic ring of the $(Z)$-styryl moiety seems to play an important role in this class of compounds. Compounds $\mathbf{2}$ and $\mathbf{3}$ showed the best potency against the CEM-13 cell lines, with $\mathrm{CTD}_{50}$ values ranging from 4.9 to $5.1 \mu \mathrm{M}$. In comparison with CA-4, all synthesized compounds presented moderate cytotoxic activity to the T-cellular human leucosis cells MT-4 and lymphoblastoid leukemia cells CEM-13, but most of them were active in the human monocyte cell lines U-937.
\end{abstract}

Keywords: furocoumarins; oreoselone; psoralen; Sonogashira coupling; semi-hydrogenation; combretastatins; cytotoxicity 


\section{Introduction}

Combretastatin A-4 (CA-4, 1, Figure 1), isolated from the bark of South African tree Combretum caffrum [1] is one of the well-known natural tubulin-binding molecules affecting microtubule dynamics by binding to the colchicine site [2]. CA-4 (1) shows strong cytotoxic activity against a wide variety of human cancer cell lines, including those that are multidrug resistant [2]. A water soluble disodium phosphate derivative of CA-4 (CA-4P, fosbretabulin) has shown promising results in human cancer clinical trials $[3,4]$, thus stimulating significant interest in a variety of CA-4 analogues [5].

Figure 1. Structures of combretastatin A-4 (1) and furocoumarin analogs of combretastatins 2-13.
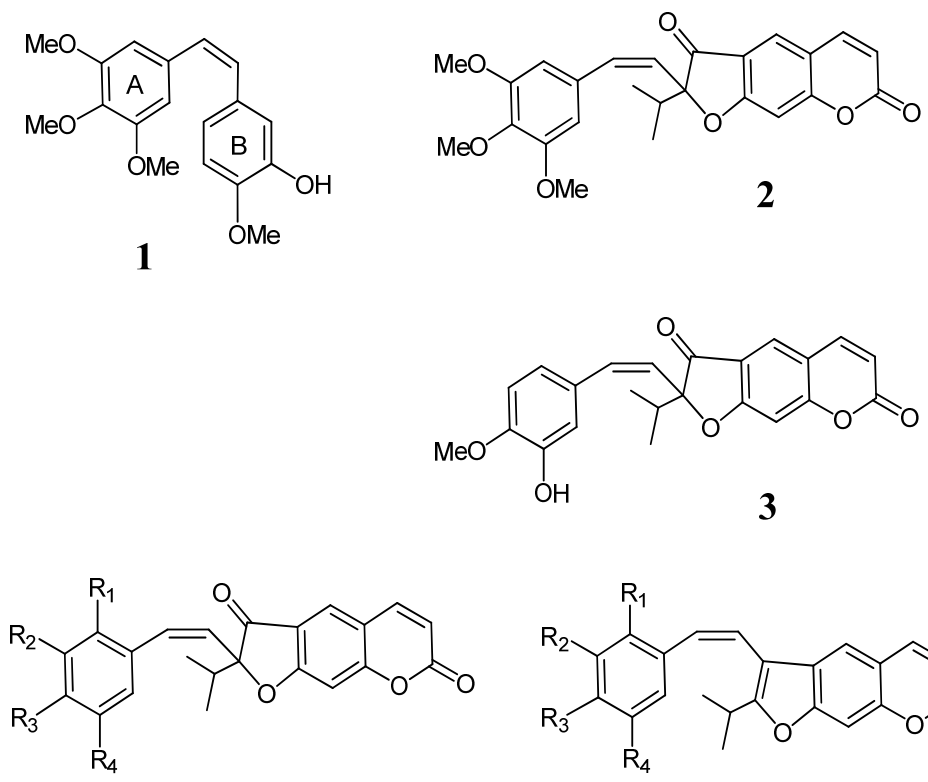

6-8<smiles>[R]c1cc(/C=C\c2c(C(C)C)oc3cc4oc(=O)ccc4cc23)c([R8])c([R])c1[R]</smiles>

9-11<smiles>COc1ccc(/C=C\c2c(C(C)C)oc3cc4oc(=O)ccc4cc23)cc1O</smiles>

5

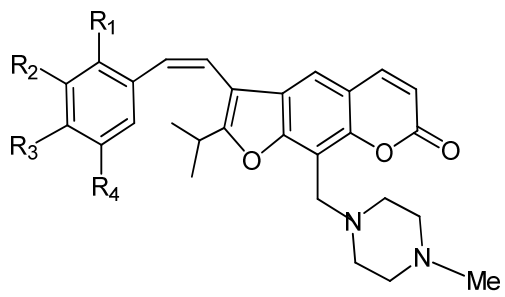

12,13

6,9 $\mathrm{R}_{1}=\mathrm{R}_{2}=\mathrm{R}_{3}=\mathrm{OMe}, \mathrm{R}_{4}=\mathrm{H}$

7,10,12 $\mathrm{R}_{1}=\mathrm{R}_{4}=\mathrm{H}, \mathrm{R}_{2}=\mathrm{R}_{3}=\mathrm{OH}$

$\mathbf{8 , 1 1 , 1 3} \mathrm{R}_{1}=\mathrm{OH}, \mathrm{R}_{2}=\mathrm{OMe}, \mathrm{R}_{3}=\mathrm{H}, \mathrm{R}_{4}=\mathrm{CHO}$<smiles>COc1cc(/C=C\c2c(C(C)C)oc3cc4oc(=O)ccc4cc23)cc(OC)c1OC</smiles>

4

Different analogs of combretastatins are obtained, including heterocombretastatins containing heterocyclic fragments as rings A or B [6-12]. A considerable cytotoxicity and antitubulin activity was revealed for heterocombretastatins containing a (4-methoxypyridin-3-yl)- or a (1-methyl-2-oxo-1,2-dihydropyridin-4-yl) ring B [6]. Compounds with benzo[b]furan ring B [9,10] or A [10], attached to the 6 position, demonstrated high antitumor activity in in vitro and in vivo models. In more recent works, replacement of the B-ring of CA-4 (1) with a (benzofuran-2-yl) moiety was also examined and the activity of the benzofuranyl derivatives was evaluated [11,12].

Herein we describe the first synthesis of combretastatin A-4 analogues $\mathbf{2 , 3}$ and $\mathbf{4 , 5}$ by replacement of the A or B aromatic ring with linear dihydrofurocoumarins or furocoumarins (psoralens) (Figure 1). The $(Z)$-styryl moiety was attached to 2- or 3-position of the heterocyclic molecule. We also include other examples of $(Z)$-arylvinylfurocoumarins 6-11 for obtaining structure-cytotoxicity relationships.

Linear furocoumarins are employed in Psoralen + UVA (PUVA) therapy for the treatment of autoimmune or hyper-proliferative skin diseases, including psoriasis and vitiligo. Activated by UV-A 
light furocoumarins induce many biological effects, such as photocycloadditions to DNA, immune system modulation, reactions with proteins, RNA and lipids [13-15]. In last decades many new potential therapeutic applications for furocoumarins are found. For instance, some psoralen derivatives were found to induce erythroid differentiation in different cellular models [16,17]. Moreover it was shown that annelation of a cyclopentane, cyclohexane, benzene, or pyridazine ring to the furan ring in the psoralen skeleton changed the phototoxicity and, in some cases led to a marked increase in the photo-antiproliferative activity [18]. There was therefore value in a targeted preparation and investigation of novel furocoumarins, containing a (Z)-styryl substituent in the furan ring of the molecules. Compounds 12 and 13, containing an additional N-methylpiperazinyl substituent in the furocoumarin scaffold were also prepared and investigated, since modification in the 9-position has a great influence on the properties of psoralenes as enzymatic inhibition and human keratinocyte proliferation [13].

\section{Results and Discussion}

\subsection{Chemical Synthesis}

The synthetic route followed for the synthesis of the desired novel combretastatin A-4 analogs 2-3, and 6-8 is outlined in Scheme 1. Previously we found that the plant coumarin oreoselone (14) by reaction with $p$-toluenesulfonyl chloride gave 2-(tosyl)oreoselone (15), which showed a high activity in Pd-catalyzed desulfonative cross-coupling reactions [19]. Herein, oreoselone (14) was converted into 2-(arylethynyl)furocoumarins 16-20 by the copper-free cross-coupling reaction with arylalkynes 21a-e in the presence of $p$-toluenesulfonyl chloride. After the purification by column chromatography compounds 16-20 were isolated in 44\%-62\% yield. 2-(Propan-2-ylidene)-7H-furo[3,2-g]chromene3,7(2H)-dione (22) [20] was also isolated with 10\%-25\% yield, presumably after elimination of $p$-toluenesulfinic acid from the in situ formed 2-(tosyl)oreoselone (15). Alkynes 16 and 20 were also prepared by the cross-coupling reaction of previously synthesized 2-(tosyl)oreoselone (15) with arylalkynes 21a,e in a THF solution in the presence of a base and a catalytic amounts of trans-dichlorobis(triphenylphosphine)palladium(II) (yield 59\%-66\%). Additionally, compound 22 was also isolated in the yield 5\%-7\%. To obtain heterocombretastatins containing a furocoumarin residue, we have studied the reduction of 2-(arylethynyl)furocoumarins 16-20. Several protocols (alkyne hydroboration [21], hydrolysis of a preformed Ti(II)-diarylalkyne complexes [22], or Lindlar's semi-hydrogenation [23]) are employed for the selective transformation of diarylalkynes into cis alkenes in the synthesis of CA-4 1. Treatment of alkynes 16, 18 with Ti(O- $i \operatorname{Pr})_{4}$ (2 equiv) and $n$-BuLi (4 equiv.) at $-78{ }^{\circ} \mathrm{C}$ in $\mathrm{THF}$ and then increasing the temperature to $50{ }^{\circ} \mathrm{C}$ and keeping the reaction mixture at this temperature for $2 \mathrm{~h}$ (for hydrolysis of the formed complexes) afforded 2-(Z)-(styryl)furocoumarins 2, 6 (yield 40\%-42\%). 2-(Z)-(Styryl)furocoumarins $\mathbf{3}, 7$ and $\mathbf{8}$ were obtained by partial hydrogenation of alkynes 17, 19 and 20 (isolated in 32\%, 24\% and 37\% yield, respectively, after column chromatography) using Lindlar's catalyst (Scheme 1). 
Scheme 1. Synthesis of 2-(Z)-styrylfurocoumarins $2,3,6,7,8$.

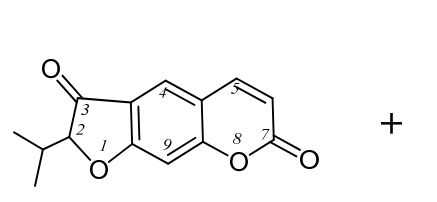

14<smiles>[R]c1cc(C#C)c([R])c([R])c1[R]</smiles>

21a-e<smiles>[R]c1cc(C=C)c([R])c([R])c1[R]</smiles>

16-20

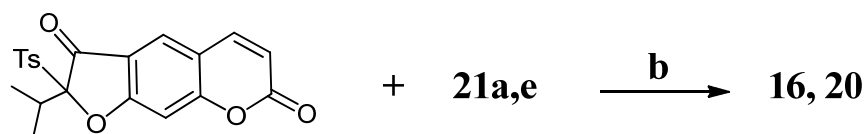

15

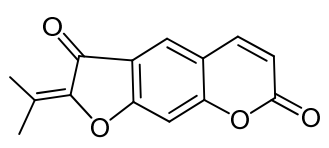

22
16,18

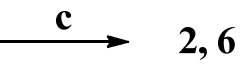
16, 21a $\mathrm{R}_{1}=\mathrm{H}, \mathrm{R}_{2}=\mathrm{R}_{3}=\mathrm{R}_{4}=\mathrm{OMe}$
17, 21b $\mathrm{R}_{1}=\mathrm{R}_{4}=\mathrm{H}, \mathrm{R}_{2}=\mathrm{OH}, \mathrm{R}_{3}=\mathrm{OMe}$
18, 21c $\mathrm{R}_{1}=\mathrm{R}_{2}=\mathrm{R}_{3}=$ OMe, $\mathrm{R}_{4}=\mathrm{H}$
$17,19,20$

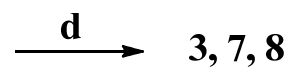
19, 21d $R_{1}=R^{4}=H, R^{2}=R^{3}=O H$
20, 21e $\mathrm{R}_{1}=\mathrm{OH}, \mathrm{R}_{2}=\mathrm{OMe}, \mathrm{R}_{3}=\mathrm{H}, \mathrm{R}_{4}=\mathrm{CHO}$

Reagents and Reaction Conditions: (a): oreoselone (14), TsCl, $\mathrm{Pd}\left(\mathrm{PPh}_{3}\right)_{2} \mathrm{Cl}_{2}(5 \mathrm{~mol} \%)$, THF, reflux, $6 \mathrm{~h}$, then alkyne 21, $\mathrm{Et}_{3} \mathrm{~N}$, THF, reflux, 7-8 h; (b): 2-tosyloreoselone (15), alkynes 21a,e, $\mathrm{Pd}_{(}\left(\mathrm{PPh}_{3}\right)_{2} \mathrm{Cl}_{2}$ $(10 \mathrm{~mol} \%), \mathrm{Et}_{3} \mathrm{~N}$, THF, reflux, $8 \mathrm{~h}$; (c) Ti(O-iPr) $)_{4}-\mathrm{n}-\mathrm{BuLi}$, THF, $-78{ }^{\circ} \mathrm{C}$, then $50{ }^{\circ} \mathrm{C}, 2 \mathrm{~h} ;(\mathbf{d}): \mathrm{H}_{2}, 1 \mathrm{~atm}$, Lindlar's catalyst, EtOH, rt, 20-30 h.

3-(Z)-(Styryl)furocoumarins 9, 10, 11, 12 and 13 (Scheme 2) are obtained from 2-isopropyl-3(trifluoromethanesulfonyloxy)psoralene (23) [24] or 2-isopropyl-9-(4-methylpiperazin-1-ylmethyl)-3(trifluoromethanesulfonyloxy)psoralene (24) [25], by approaches described early for preparation of compounds 4,5 [26]. The reaction of triflates 23 with arylalkynes $21 \mathbf{c}-\mathbf{e}$ in a benzene (WARNINGthis solvent is a known carcinogenic substance) solution in the presence of a catalytic amounts of trans-dichlorobis(triphenylphosphine)palladium(II), copper(I) iodide, and triethylamine led to the corresponding 3-(arylalkyne)furocoumarins 25-27 in the yield 68\%-73\% after the column chromatography. By using the mentioned cross-coupling conditions for reacting of psoralen derivatives 24 with the alkynes 21d,e, compounds 28, 29 are obtained (yield 42\%-58\%). For preparation of compound $\mathbf{2 6}$ we employed another approach. At first, by the Sonogashira coupling of oreoselone triflate $\mathbf{2 3}$ with (trimethylsilyl)acetylene $\mathbf{3 0}$ we obtained 3-(trimethylsilyl)-2-isopropylpsoralene (31) (yield 58\%). Desilylation of compound 31 produced the corresponding 3-ethynyl-2isopropylpsoralen (32) (66\% yield). Hydrolysis of in situ formed Ti(II)-alkyne complex of alkyne 25 gave the 3-(Z)-(2,3,4-trimethoxystyryl)furocoumarin (9) (yield 60\%). Partial reduction of alkynes 26-29 using Lindlar's catalyst resulted in the isolation of 3-(Z)-styrylsubstituted furocoumarins 10, 11, 12, or 13 (yield $40 \%-60 \%$ ) (Scheme 2). 
Scheme 2. Synthesis of 3-(Z)-styrylfurocoumarins 9-13.<smiles>CC(C)c1oc2cc3oc(=O)ccc3cc2c1O</smiles>

23<smiles>[R]c1cc(C#C)c([R])c([R])c1[R]</smiles>

21c-e
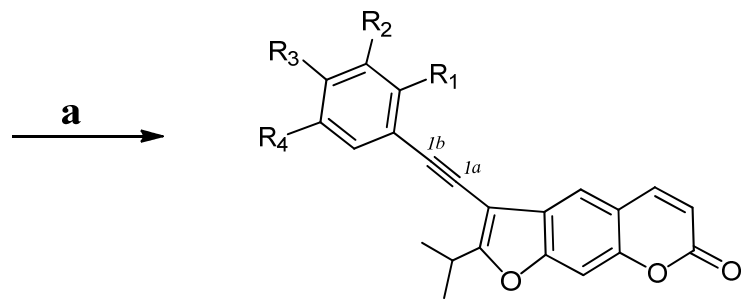

25-27

21c, $25 \mathrm{R}_{1}=\mathrm{R}_{2}=\mathrm{R}_{3}=\mathrm{OMe}, \mathrm{R}_{4}=\mathrm{H}$

21d, $26 \mathrm{R}_{1}=\mathrm{R}_{4}=\mathrm{H}, \mathrm{R}_{2}=\mathrm{R}_{3}=\mathrm{OH}$

21e, $27 \mathrm{R}_{1}=\mathrm{OH}, \mathrm{R}_{2}=\mathrm{OMe}, \mathrm{R}_{3}=\mathrm{H}, \mathrm{R}_{4}=\mathrm{CHO}$<smiles>CCCOc1c(C(C)C)oc2c(CN3CCN(C)CC3)c3oc(=O)ccc3cc12</smiles>

24

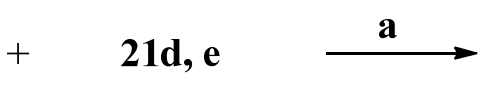

$28 \mathrm{R}_{1}=\mathrm{R}_{4}=\mathrm{H}, \mathrm{R}_{2}=\mathrm{R}_{3}=\mathrm{OH}$

$29 \mathrm{R}_{1}=\mathrm{OH}, \mathrm{R}_{2}=\mathrm{OMe}, \mathrm{R}_{3}=\mathrm{H}, \mathrm{R}_{4}=\mathrm{CHO}$

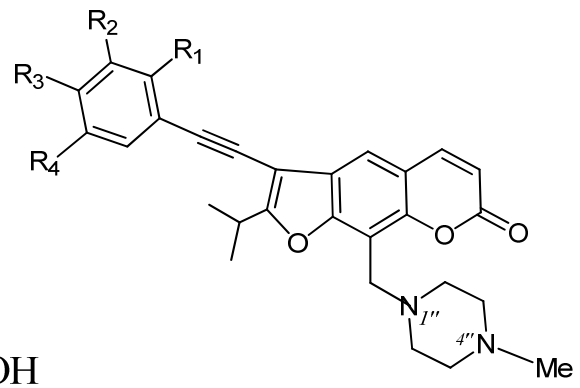

28, 29

23

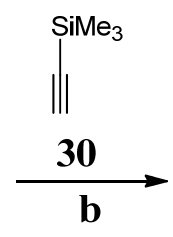

25 $\mathbf{e} \rightarrow \mathbf{9}$<smiles>C#Cc1c(C(C)C)oc2cc3oc(=O)ccc3cc12</smiles>

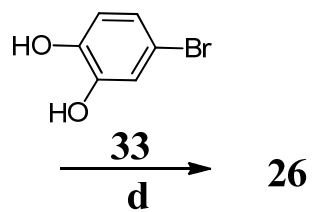

32

Reagents and Reaction Conditions: (a): triflate 23, or 24, $\mathrm{Pd}\left(\mathrm{PPh}_{3}\right)_{2} \mathrm{Cl}_{2}(4 \mathrm{~mol} \%), \mathrm{CuI}\left(2 \mathrm{~mol}_{0}\right), \mathrm{Et}_{3} \mathrm{~N}$, alkynes 21c-e, $\mathrm{PhH}$, reflux, 8-10 h; (b): triflate 23, alkyne 30, $\mathrm{Pd}\left(\mathrm{PPh}_{3}\right)_{2} \mathrm{Cl}_{2}(4 \mathrm{~mol} \%), \mathrm{CuI}\left(2 \mathrm{~mol}_{\%}\right), \mathrm{Et}_{3} \mathrm{~N}$, $\mathrm{PhH}$, reflux, 10 h; (c): CsF, MeOH, benzyltrimethylammonium chloride, rt; (d): $\left.\mathrm{Pd}_{(} \mathrm{PPh}_{3}\right)_{2} \mathrm{Cl}_{2}(4 \mathrm{~mol} \%), \mathrm{CuI}$ (2 mol \%), $\mathrm{Et}_{3} \mathrm{~N}$, bromide 33, $\mathrm{PhH}$, reflux, $10 \mathrm{~h}$; (e): $\mathrm{Ti}(\mathrm{O}-i \mathrm{Pr})_{4}-n-\mathrm{BuLi}, \mathrm{THF},-78{ }^{\circ} \mathrm{C}$, then $50{ }^{\circ} \mathrm{C}, 2 \mathrm{~h} ;(\mathbf{f}): \mathrm{H}_{2}$, 1 atm, Lindlar's catalyst, EtOH, rt, 30 h.

The structure of the synthesized compounds was established based on the combination of IR, UV, and NMR spectral data. The IR spectra of compounds 16-20, 25, 26, 27, 28, 29, 31 and 32 is characterized by the presence of the absorption band of the alkyne linker group at 2046-2191 cm ${ }^{-1}$. The ${ }^{1} \mathrm{H}$ and ${ }^{13} \mathrm{C}-\mathrm{NMR}$ spectra of all synthesized compounds agree well with their structure and contain one set of characteristic signals of psoralen (3-oxo-2,3-dihydrofurocoumarin) skeleton and the corresponding substituent. The spin-spin coupling constants between alkenyl proton signals $\mathrm{H}-1 \mathrm{a}$ and $\mathrm{H}-1 \mathrm{~b}$ $(J 8.8-9.5 \mathrm{~Hz})$ indicate the formation of compounds $\mathbf{2}-\mathbf{1 3}$ with the $(Z)$-configuration of the double bond. 


\subsection{Cytotoxicity Studies}

The cytotoxic activity of the synthesized series of 2-(Z)-styryldihydrofurocoumarins $\mathbf{2 , 3 , 6 - 8}$, 3-(Z)-styrylfurocoumarins 4,5,9-13, the parent oreoselone (14) and combretastatin A-4 (1) was determined by measuring the concentration inhibiting human tumor cell viability by $50 \%\left(\mathrm{CTD}_{50}\right)$. The $\mathrm{CTD}_{50}$ was determined using the conventional MTT assay, which allows to estimate the number of survived cells spectrophotometrically [27]. The results are presented in Table 1. At first glance, it is evident that there is a difference in activity between the parent compound $\mathbf{1 4}$ and $(Z)$-styryl modified furocoumarins 2-13.

Table 1. Cytotoxic activity of heterocyclic analogs of combretastatins 2-13.

\begin{tabular}{cccc}
\hline \multirow{2}{*}{ Compound } & \multicolumn{3}{c}{ Cytotoxicity $\left(\mathbf{C T D}_{\mathbf{5 0}}, \boldsymbol{\mu M}\right)$ against Cell Line ${ }^{\boldsymbol{a}}$} \\
\cline { 2 - 4 } & $\mathbf{C E M}-\mathbf{M 3}$ & $\mathbf{M T}-\mathbf{4}$ & $\mathbf{U}_{\mathbf{9 3}}$ \\
\hline 2 & $4.9 \pm 0.2$ & $13.4 \pm 4.5$ & $12.1 \pm 2.0$ \\
3 & $5.1 \pm 0.9$ & $10.1 \pm 2.1$ & $18.0 \pm 2.9$ \\
4 & $28.6 \pm 2.1$ & $22.4 \pm 2.6$ & $41.3 \pm 6.8$ \\
5 & $29.0 \pm 3.2$ & $36.1 \pm 4.6$ & $32.1 \pm 3.5$ \\
6 & $22.1 \pm 2.3$ & $28.6 \pm 3.7$ & $33.1 \pm 3.9$ \\
7 & $9.3 \pm 2.6$ & $9.2 \pm 0.5$ & $12.0 \pm 0.8$ \\
8 & $14.1 \pm 1.6$ & $12.1 \pm 3.2$ & $13.3 \pm 2.1$ \\
9 & $95.0 \pm 3.4$ & $24.5 \pm 3.2$ & $>100$ \\
10 & $49.1 \pm 4.5$ & $13 \pm 2.8$ & $29.0 \pm 3.1$ \\
11 & $26.7 \pm 2.9$ & $45.1 \pm 1.9$ & $28.0 \pm 4.2$ \\
12 & $20.2 \pm 1.6$ & $5.2 \pm 0.8$ & $9.1 \pm 1.2$ \\
13 & $9.0 \pm 2.6$ & $8.1 \pm 0.7$ & $12.1 \pm 2.3$ \\
$(14)$ & $>100$ & $70.9 \pm 3.6$ & $65.2 \pm 3.9$ \\
CA-4 (1) & $0.8 \pm 0.03$ & $0.1 \pm 0.012$ & $>100^{b}$ \\
\hline
\end{tabular}

${ }^{a}$ The cell were continuously treated with compounds for $72 \mathrm{~h} ;{ }^{b}$ CA-4 provide inhibition of $47 \%$ in concentration $0.1-100 \mu \mathrm{M}$.

All synthesized compounds $\mathbf{2 - 1 3}$ exhibited cytotoxic activity in respect to model cancer cell lines and were more potent than furocoumarin 14. Furocoumarins 4, 5, 9, 10 and 11, in which the $(Z)$-styryl substituent was located in the 3-position, demonstrated decrease of potency compared to 2-(Z)-styryl3-oxodihydrofurocoumarins $2,3,6,7$ and 8 . Compounds 2,3 possessed the best activity on the lymphoblastoid leukemia cells CEM-13. The phenolic substituent in $(Z)$-styryl moiety seems to have an important role in this class of compounds; indeed 3,4-dihydroxyphenyl substituent in compounds 7, 10, 12 demonstrated increase of potency against MT-4 cell lines. Compounds having a 2,3,4trimethoxyphenyl moiety (compounds 6, 9) show week cytotoxic activity, while several derivatives containing a 3,4,5-trimethoxyphenyl residue (compounds 2,4 ) shown improved activity. The latter effect is unsurprising, since it is well established that the replacement of A-ring in combretastatins [28,29] and phenstatin [30] is highly detrimental for the activity of the compounds. In the 3,9-disubstituted furocoumarins $\mathbf{1 2 , 1 3}$ cytotoxicity does not seem to greatly influence by the substituent in the aromatic ring, however, the (4-methylpiperazin-1-ylmethyl) substitution on the 9th-position in 3-styrylfurocoumarin core gave increase in cytotoxic activity of compounds (compare 10 and $\mathbf{1 2}$ or $\mathbf{1 1}$ and 13), especially, on the human monocyte-lines U-937. The activity of all synthesized compounds on the lymphoblastoid 
cell lines was lower than the activity of CA-4 1, however, compounds $\mathbf{2 , 7 , 1 2}$ and 13 shown potency in respect to human monocyte-lines U-937 in comparison with CA-4.

\section{Experimental Section}

\subsection{General}

NMR spectra were acquired on Bruker AV-400 ( $\left.{ }^{1} \mathrm{H}: 400.13 \mathrm{MHz},{ }^{13} \mathrm{C}: 100.78 \mathrm{MHz}\right)$ or Bruker AV-600 ( ${ }^{1} \mathrm{H}$ : $600.30 \mathrm{MHz},{ }^{13} \mathrm{C}$ : $150.95 \mathrm{MHz}$ ) (Bruker BioSpin GmbH, Rheinstetten, Germany) instruments, using tetramethylsilane (TMS) as an internal standart. In the description of the ${ }^{1} \mathrm{H}$ and ${ }^{13} \mathrm{C}$-NMR spectra, the furocoumarin skeleton atoms numeration system given in structure $\mathbf{1 4}$ was used. The IR spectra were recorded by means of the $\mathrm{KBr}$ pellet technique on a Bruker Vector-22 spectrometer. The UV spectra were obtained on an HP $8453 \mathrm{UV}-\mathrm{V}$ is spectrometer (Hewlett-Packard, Waldbronn, Germany). The melting points were determined on a Stuart SMF-38 melting point apparatus (Bibby Scientific, Staffordshire, UK) and are uncorrected. Elemental analysis was carried out on a Carlo-Erba 1106 Elemental analysis instrument (Carlo-Erba, Milan, Italy). Spectral and analytical investigations were carried out at Collective Chemical Service center of Siberian Branch of the Russian Academy of Sciences.

Reaction products were isolated by column chromatography on silica gel $60(0.063-0.200 \mathrm{~mm}$, Merck KGaA, Darmstadt, Germany) and eluted with chloroform and chloroform-ethanol (100:1; to 25:1). The reaction progress and the purity of the obtained compounds were monitored by TLC on Silufol UV-254 plates (Kavalier, Czech Republic, $\mathrm{CHCl}_{3}$-EtOH, 25:1; detection under UV light or by treatment with iodine vapor).

Chemicals used-TsCl, Ti(O- $i \mathrm{Pr})_{4}, \mathrm{n}-\mathrm{BuLi}, \mathrm{CuI}, \mathrm{PPh}_{3}$, Lindlar's catalysts and (trimethylsilyl)acetylene 30 -were purchased from Sigma-Aldrich (St. Louis, MO, USA) or Alfa Aesar (GmbH, Karlsruhe, Germany). Dichlorobis(triphenylphosphine)palladium(II) was obtained as described in [31]. 1-Ethynyl-3,4,5-trimethoxybenzene (21a) [32], 1-ethynyl-3-hydroxy-4-trimethoxybenzene (21b) [33], 1-ethynyl-2,3,4-trimethoxybenzene (21c) [34], 1-ethynyl-3,4-dihydroxybenzene (21d) [35], 1-ethynyl2-hydroxy-3-methoxy-5-formylbenzene (21e) [36], 3,4-dihydroxybromobenzene (33) [37] and combretastatin A-4 (1) [22] are known compounds and were prepared by the reported methods. Solvents (THF, benzene, $\mathrm{MeOH}$ ) and $\mathrm{Et}_{3} \mathrm{~N}$ were purified by standard methods and distilled in a stream of argon just before use.

\subsection{Synthesis and Spectral Data}

2-Isopropyl-2-[(3,4,5-trimethoxyphenyl)ethynyl]-2H-furo[3,2-g]chromene-3,7-dione (16). (a) To a solution of oreoselone $(\mathbf{1 4}, 488 \mathrm{mg}, 2 \mathrm{mmol})$ in THF $(7 \mathrm{~mL})$ under argon was added $\mathrm{TsCl}$ (470 mg, $2.5 \mathrm{mmol})$ and $\mathrm{Pd}\left(\mathrm{PPh}_{3}\right)_{2} \mathrm{Cl}_{2}(7 \mathrm{mg}, 0.1 \mathrm{mmol})$. The mixture was heated at $60{ }^{\circ} \mathrm{C}$ for $6 \mathrm{~h}$ (TLC), then 1-ethynyl-3,4,5-trimethoxybenzene (21a, $770 \mathrm{mg}, 4 \mathrm{mmol})$ and $\mathrm{Et}_{3} \mathrm{~N}(0.84 \mathrm{~mL}, 6 \mathrm{mmol})$ was added. The reaction mixture was heated under stirring another $7 \mathrm{~h}$. After cooling, $3 \mathrm{~mL}$ of water was added and the mixture was extracted with methylene chloride $(5 \times 4 \mathrm{~mL})$. The combined extract was washed with water, dried over $\mathrm{MgSO}_{4}$ and filtered. The solvent was removed under reduced pressure, and the residue was subjected to column chromatography to isolate $538 \mathrm{mg}(62 \%)$ of 16 and 
$40 \mathrm{mg}(12 \%)$ of 22. Compound 16 was recrystallized from diethyl ether, m.p. $101-102{ }^{\circ} \mathrm{C}$. IR (KBr, $v$, $\mathrm{cm}^{-1}$ ): 2974, 2927, 2879, 2852, 2150, 1732, 1664, 1626, 1531, 1481, 1411, 1390, 1353, 1286, 1253, 1126, 1095, 1039, 970, 948, 916, 866, 827, 734, 661. ${ }^{1} \mathrm{H}-\mathrm{NMR}\left(600 \mathrm{MHz}, \mathrm{CDCl}_{3}, \delta_{\mathrm{H}}\right): 0.98(\mathrm{~d}, J=6.9 \mathrm{~Hz}$, $\left.3 \mathrm{H}, \mathrm{CH}_{3}\right), 1.28\left(\mathrm{~d}, J=6.9 \mathrm{~Hz}, 3 \mathrm{H}, \mathrm{CH}_{3}\right), 2.46\left[\mathrm{~m}, 1 \mathrm{H}, \mathrm{C} \underline{\mathrm{H}}-\left(\mathrm{CH}_{3}\right)_{2}\right], 3.84\left(\mathrm{~s}, 9 \mathrm{H}, 3 \times \mathrm{OCH}_{3}\right), 6.34(\mathrm{~d}$, $J=9.6 \mathrm{~Hz}, 1 \mathrm{H}, \mathrm{H}-6), 6.96$ (s, 1H, H-9), 7.04 (s, 2H, H-2',6'), 7.68 (d, $J=9.6 \mathrm{~Hz}, 1 \mathrm{H}, \mathrm{H}-5), 7.87$ (s, $1 \mathrm{H}, \mathrm{H}-4) .{ }^{13} \mathrm{C}-\mathrm{NMR}\left(150 \mathrm{MHz}, \mathrm{CDCl}_{3}, \delta_{\mathrm{C}}\right): 16.5\left(\mathrm{CH}_{3}\right), 17.4\left(\mathrm{CH}_{3}\right), 36.4(\mathrm{CH}), 56.0\left(3 \mathrm{C}-\mathrm{CH}_{3}\right)$, 72.1 (C-1a), 82.6 (C-1b), 99 (C-2), 101.4 (C-9), 106.5 (C-2' and C-6'), 115.2 (C-6), 115.6, 115.5 (C-3a,4a),119.4 (C-1'), 125.8 (C-4), 131.5 (C-4'), 143.0 (C-5), 153.4 (C-8a), 155.8 (C-3' and C-5'), 158.6 (C-7), 169.4 (C-9a), 193.8 (C-3). UV (EtOH) $\lambda_{\max }$, (lge): 257 (4.56), 297 (4.3), 307 (4.27), 348 (4.11) nm. Anal. Calcd for $\mathrm{C}_{25} \mathrm{H}_{22} \mathrm{O}_{7}$ : C, 69.12; H, 5.10; found $\mathrm{C}, 69.29 ; \mathrm{H}, 5.02$. (b) A mixture of 2-( $p$-toluenesulfonyl)oreoselone (15, $398 \mathrm{mg}, 1 \mathrm{mmol})$, 1-ethynyl-3,4,5-trimethoxybenzene (21a, $385 \mathrm{mg}, 2 \mathrm{mmol}), \mathrm{Et}_{3} \mathrm{~N}(0.42 \mathrm{~mL}, 3 \mathrm{mmol})$, and $\mathrm{Pd}\left(\mathrm{PPh}_{3}\right)_{2} \mathrm{Cl}_{2}(7 \mathrm{mg}, 0.1 \mathrm{mmol})$ in $5 \mathrm{~mL}$ of anhydrous THF was heated for $7 \mathrm{~h}$ under reflux in argon. The mixture was evaporated, the residue was treated with $10 \mathrm{~mL}$ of water and extracted with methylene chloride $(4 \times 5 \mathrm{~mL})$. The combined extract was dried over $\mathrm{MgSO}_{4}$, filtered and evaporated. The residue was subjected to column chromatography on silica gel to isolate $287 \mathrm{mg}$ (66\%) of compound 16 and $12 \mathrm{mg}$ (5\%) of compound 22.

2-[(3-Hydroxy-4-methoxyphenyl)ethynyl]-2-isopropyl-2H-furo[3,2-g]chromene-3,7-dione (17). This compound (374 mg, 48\%) was prepared as a yellow oil from oreoselone (14, $488 \mathrm{mg}, 2 \mathrm{mmol})$ and 1-ethynyl-3-hydroxy-4-methoxybenzene (21b (592 $\mathrm{mg}, 4 \mathrm{mmol})$ using the procedure $(a)$ described for 16. IR (KBr, $\left.v, \mathrm{~cm}^{-1}\right)$ : 3467, 3086, 3062, 3030, 2976, 2923, 2850, 2191, 1751, 1660, 1625, 1600, 1494, 1419, 1353, 1267, 1207, 1149, 1110, 1066, 1028, 977, 935, 806, 752, 696. ${ }^{1} \mathrm{H}-\mathrm{NMR}(400 \mathrm{MHz}$, $\left.\mathrm{CDCl}_{3}, \delta_{\mathrm{H}}\right): 1.09\left(\mathrm{~d}, J=6.9 \mathrm{~Hz}, 3 \mathrm{H}, \mathrm{CH}_{3}\right), 1.20\left(\mathrm{~d}, J=6.9 \mathrm{~Hz}, 3 \mathrm{H}, \mathrm{CH}_{3}\right), 3.03\left[\mathrm{~m}, 1 \mathrm{H}, \mathrm{C} \underline{\mathrm{H}}-\left(\mathrm{CH}_{3}\right)_{2}\right]$, $3.79\left(\mathrm{~s}, 3 \mathrm{H}, \mathrm{OCH}_{3}\right), 6.05(\mathrm{~s}, 1 \mathrm{H}, \mathrm{OH}), 6.40$ (d, J=9.6 Hz, 1H, H-6), 6.92 (d, J = $\left.7.8 \mathrm{~Hz}, 1 \mathrm{H}, \mathrm{H}-5^{\prime}\right)$, 6.97 (dd, $J=7.8$ and $\left.1.8 \mathrm{~Hz}, 1 \mathrm{H}, \mathrm{H}-6^{\prime}\right), 6.98$ (s, 1H, H-9), 6.99 (d, $\left.J=1.8 \mathrm{~Hz}, 1 \mathrm{H}, \mathrm{H}-2^{\prime}\right), 7.66$ (d, $J=9.6 \mathrm{~Hz}, 1 \mathrm{H}, \mathrm{H}-5), 7.85$ (s, $1 \mathrm{H}, \mathrm{H}-4) .{ }^{13} \mathrm{C}-\mathrm{NMR}\left(100 \mathrm{MHz}, \mathrm{CDCl}_{3}, \delta_{\mathrm{C}}\right): 16.2\left(\mathrm{CH}_{3}\right), 17.4\left(\mathrm{CH}_{3}\right)$, $30.1(\mathrm{CH}), 54.1\left(\mathrm{CH}_{3}\right), 73.5(\mathrm{C}-1 \mathrm{a}), 82.4(\mathrm{C}-1 \mathrm{~b}), 96.1(\mathrm{C}-2), 100.1$ (C-9), $112.4(\mathrm{C}-6), 113.1\left(\mathrm{C}-5{ }^{\prime}\right)$, 113.4 (C-3a), 115.2 (C-4a), 118.1 (C-1'), 120.5 (C-2'), 123.1 (C-6'), 125.7 (C-4), 142.3 (C-5), 147.9 (C-3'), 149.1 (C-4'), 156.6 (C-8a), 158.1 (C-7), 172.6 (C-9a), 195.5 (C-3). UV (EtOH) $\lambda_{\max }(\lg \varepsilon)$ : 258 (4.52), 295 (3.96), 307 (3.88), 351 (3.93) nm. Anal. Calcd for $\mathrm{C}_{23} \mathrm{H}_{18} \mathrm{O}_{6}$ : C, 70.76; H, 4.65; found C, 70.39; H, 4.92 .

2-Isopropyl-2-[(2,3,4-trimethoxyphenyl)ethynyl]-2H-furo[3,2-g]chromene-3,7-dione (18). Compound 18 (560 mg, 65\%) was prepared from oreoselone (14, $488 \mathrm{mg}, 2 \mathrm{mmol})$ and 1-ethynyl-2,3,4trimethoxybenzene (21c, $770 \mathrm{mg}, 4 \mathrm{mmol}$ ) using the procedure (a) described for 16. Compound 22 (30 $\mathrm{mg}$, $10 \%$ was also isolated. Compound 18, m.p. $101-102{ }^{\circ} \mathrm{C}$ (ether). IR (KBr, v, cm $\left.{ }^{-1}\right): 3066,2976,2939$, 2861, 2189, 1737, 1679, 1625, 1487, 1423, 1388, 1352, 1286, 1224, 1141, 1122, 1093, 1022, 983, 943, 866, 825, 790, 734, 691. ${ }^{1} \mathrm{H}-\mathrm{NMR}\left(400 \mathrm{MHz}, \mathrm{CDCl}_{3}, \delta_{\mathrm{H}}\right): 0.91\left(\mathrm{~d}, J=6.9 \mathrm{~Hz}, 3 \mathrm{H}, \mathrm{CH}_{3}\right), 1.31(\mathrm{~d}, J=$ $\left.6.9 \mathrm{~Hz}, 3 \mathrm{H}, \mathrm{CH}_{3}\right), 2.50\left[\mathrm{~m}, 1 \mathrm{H}, \mathrm{C} \underline{\mathrm{H}}-\left(\mathrm{CH}_{3}\right)_{2}\right], 3.82\left(\mathrm{~s}, 3 \mathrm{H}, \mathrm{OCH}_{3}\right), 3.88\left(\mathrm{~s}, 3 \mathrm{H}, \mathrm{OCH}_{3}\right), 3.97(\mathrm{~s}, 3 \mathrm{H}$, $\left.\mathrm{OCH}_{3}\right), 6.34$ (d, $\left.J=9.6 \mathrm{~Hz}, 1 \mathrm{H}, \mathrm{H}-6\right), 6.70$ (d, $J=8.2 \mathrm{~Hz}, 1 \mathrm{H}, \mathrm{H}-5$ '), 6.99 (s, 1H, H-9), 7.52 (d, 1H, $J=$ $8.2 \mathrm{~Hz}, \mathrm{H}-6$ '), 7.72 (d, 1H, $J=9.6 \mathrm{~Hz}, \mathrm{H}-5), 7.90(1 \mathrm{H}, \mathrm{s}, \mathrm{H}-4) .{ }^{13} \mathrm{C}-\mathrm{NMR}\left(100 \mathrm{MHz}, \mathrm{CDCl}_{3}, \delta_{\mathrm{C}}\right): 16.6$ $\left(\mathrm{CH}_{3}\right), 17.5\left(\mathrm{CH}_{3}\right), 36.4(\mathrm{CH}), 56.0\left(\mathrm{CH}_{3}\right), 60.8\left(\mathrm{CH}_{3}\right), 62.2\left(\mathrm{CH}_{3}\right), 71.6(\mathrm{C}-1 \mathrm{a}), 82.3(\mathrm{C}-1 \mathrm{~b}), 98.9(\mathrm{C}-$ 
2), 101.5 (C-9), 107.2 (C-5'), 115.4 (C-3a,4a), 115.3 (C-6), 115.6, 123.1 (C-1'), 124.0 (C-6'), 125.9 (C4), 141.4 (C-3'), 143.1 (C-5), 156.7 (C-2'), 158.8 (C-4'), 159.1 (C-8a),161.1 (C-7), 169.5 (C-9a), 192.6 (C-3). UV (EtOH) $\lambda_{\max }(\lg \varepsilon): 257$ (4.42), 297 (4.17), 307 (4.14), 348 (3.97) nm. Anal. Calcd for $\mathrm{C}_{25} \mathrm{H}_{22} \mathrm{O}_{7}$ : C, 69.12; $\mathrm{H}, 5.10$; found $\mathrm{C}, 69.49 ; \mathrm{H}, 5.12$.

2-[(3,4-Dihydroxyphenyl)ethynyl]-2-isopropyl-2H-furo[3,2-g]chromene-3,7-dione (19). Compound 19 (330 mg, 44\%) was prepared from oreoselone (14, $488 \mathrm{mg}, 2 \mathrm{mmol}$ ) and 1-ethynyl-2,3,4trimethoxybenzene (21d, $540 \mathrm{mg}, 4 \mathrm{mmol})$ using the procedure $(a)$ described for 16. Compound 22 (82 mg, 25\%) was also isolated. Compound 19, m.p. 84-86 ${ }^{\circ} \mathrm{C}$ (ether). IR ( $\mathrm{KBr}, v, \mathrm{~cm}^{-1}$ ): 3340, 3320, 3087, 3071, 2974, 2929, 2879, 2852, 2114, 1732, 1664, 1625, 1595, 1531, 1390, 1353, 1286, 1253, 1126, 1095, 1039, 948, 866, 827, 735. ${ }^{1} \mathrm{H}-\mathrm{NMR}\left(400 \mathrm{MHz}, \mathrm{CDCl}_{3}, \delta_{\mathrm{H}}\right): 0.96\left(\mathrm{~d}, J=7.0 \mathrm{~Hz}, 3 \mathrm{H}, \mathrm{CH}_{3}\right)$, 1.37 (d, $\left.J=7.0 \mathrm{~Hz}, 3 \mathrm{H}, \underline{\mathrm{C}}_{3}\right), 2.55$ [m, 1H, $\left.\underline{\mathrm{H}}-\left(\mathrm{CH}_{3}\right)_{2}\right], 6.40$ (d, J=9.6 Hz, 1H, H-6), 7.05 (s, 1H, H-9), $7.46\left(\mathrm{~d}, J 1.8 \mathrm{~Hz}, 1 \mathrm{H}, \mathrm{H}-2^{\prime}\right), 7.52$ (dd, $J=8.0$ and $\left.1.8 \mathrm{~Hz}, 1 \mathrm{H}, \mathrm{H}-6^{\prime}\right), 7.66$ (d, $\left.J=8.0 \mathrm{~Hz}, 1 \mathrm{H}, \mathrm{H}-5^{\prime}\right)$, $7.73(\mathrm{~d}, J=9.6 \mathrm{~Hz}, 1 \mathrm{H}, \mathrm{H}-5), 7.93$ (s, $1 \mathrm{H}, \mathrm{H}-4) .{ }^{13} \mathrm{C}-\mathrm{NMR}\left(100 \mathrm{MHz}, \mathrm{CDCl}_{3}\right): 15.6\left(\mathrm{CH}_{3}\right), 16.0\left(\mathrm{CH}_{3}\right)$, $31.1(\mathrm{CH}), 73.9$ (C-1a), 81.9 (C-1b), 98.6 (C-2), 101.4 (C-9), 115.7 (C-3a), 116.0 (C-6), 116.7 (C-4a), 117.8 (C-1'), 120.2 (C-5'), 122.7 (C-2'), 124.1 (C-4), 126.1 (C-6'), 143.3 (C-5), 158.5 (C-8a), 159.3 (C-3'), 160.4 (C-4'), 161.9 (C-7), 171.8 (C-9a), 191.9 (C-3). UV (EtOH) $\lambda_{\max }$ (lge): 256 (4.4), 303 (3.92), 348 (3.87) nm. Anal. Calcd for $\mathrm{C}_{22} \mathrm{H}_{16} \mathrm{O}_{6}$ : C, 70.21; H, 4.29; found C, 70.49; H, 4.20.

2-[(5-Formyl-3-hydroxy-3-methoxyphenyl)ethynyl]-2-isopropyl-2H-furo[3,2-g]chromene-3,7-dione

(20). Compound 20 (493 mg, 59\%) was prepared from oreoselone (14, $488 \mathrm{mg}, 2 \mathrm{mmol}$ ) and 1-ethynyl-5-formyl-2-hydroxy-3-methoxybenzene (21e, $705 \mathrm{mg}, 4 \mathrm{mmol}$ ) using the procedure $(a)$ described for 16, or from 2-( $p$-toluenesulfonyl)oreoselone $(\mathbf{1 5}, 398 \mathrm{mg}, 1 \mathrm{mmol})$ and 1-ethynyl-5formyl-2-hydroxy-3-methoxybenzene (21e, $353 \mathrm{mg}, 2 \mathrm{mmol}$ ) using the procedure (b) described for 16. Yield 60\%. Compound 22 was also isolated [62 $\mathrm{mg}$, procedure $(a)$ and $23 \mathrm{mg}$, procedure $(b)$ ]. Compound 20, m.p. $91-93{ }^{\circ} \mathrm{C}$ (ether). IR (KBr, v, $\mathrm{cm}^{-1}$ ): 3456, 3155, 3065, 2978, 2939, 2881, 2839, 2112, 1738, 1680, 1626, 1582, 1554, 1487, 1423, 1389, 1352, 1286, 1260, 1250, 1225, 1201, 1141, 1122, 1094, 1043, 1022, 984, 970, 943, 916, 866, 826, 790, 760, 735, 690, 6800. ${ }^{1} \mathrm{H}-\mathrm{NMR}(400 \mathrm{MHz}$, $\left.\mathrm{CDCl}_{3}, \delta_{\mathrm{H}}\right): 0.91\left(\mathrm{~d}, J=6.9 \mathrm{~Hz}, 3 \mathrm{H}, \mathrm{CH}_{3}\right), 1.29\left(\mathrm{~d}, J=6.9 \mathrm{~Hz}, 3 \mathrm{H}, \mathrm{CH}_{3}\right), 2.48$ [m, 1H, Cㅍ- $\left.\left(\mathrm{CH}_{3}\right)_{2}\right]$, $3.77\left(\mathrm{~s}, 3 \mathrm{H}, \mathrm{OCH}_{3}\right), 6.32(\mathrm{~d}, J=9.8 \mathrm{~Hz}, 1 \mathrm{H}, \mathrm{H}-6), 6.96$ (s, 1H, H-9), 7.43 (br s, 1H, H-4'), 7.50 (br s, 1H, OH), 7.60 (br s, 1H, H-6'), 7.69 (d, $J=9.8 \mathrm{~Hz}, 1 \mathrm{H}, \mathrm{H}-5), 7.88$ (s, 1H, H-4), 9.91 (s, 1H, CHO). ${ }^{13} \mathrm{C}-\mathrm{NMR}\left(100 \mathrm{MHz}, \mathrm{CDCl}_{3}, \delta_{\mathrm{C}}\right): 15.2\left(\mathrm{CH}_{3}\right), 15.6\left(\mathrm{CH}_{3}\right), 33.7(\mathrm{CH}), 55.4\left(\mathrm{OCH}_{3}\right), 71.3(\mathrm{C}-1 \mathrm{a}), 80.2$ (C-1b), 98.3 (C-2), 101.1 (C-9), 115.4 (C-3a), 115.7 (C-6), 116.4 (C-4'), 118.3 (C-4a), 119.5 (C-1'), 121.5 (C-6'), 125.7 (C-4), 133.6 (C-5'), 143.1 (C-5), 156.6 (C-3'), 158.9 (C-2'), 161.6 (C-7), 165.1 (C-8a), 171.5 (C-9a), 191.6 (CHO), 192.3 (C-3). UV (EtOH) $\lambda_{\max }$ (lge): 253 (4.21), 328 (3.45), 350 (3.15) nm. Anal. Calcd for $\mathrm{C}_{24} \mathrm{H}_{18} \mathrm{O}_{7}$ : C, 68.90; H, 4.34; found $\mathrm{C}, 68.69 ; \mathrm{H}, 4.56$.

(Z)-2-Isopropyl-2-(3,4,5-trimethoxystyryl)-2H-furo[3,2-g]chromene-3,7-dione (2). A solution of $n$-BuLi $(0.08 \mathrm{~mL}, 1.04 \mathrm{mmol})$ was added dropwise to a solution of 2-isopropyl-2-[(3,4,5trimethoxyphenyl)ethynyl]-2H-furo[3,2-g]chromene-3,7-dione $\quad(\mathbf{1 6}, 100 \mathrm{mg}, \quad 0.23 \mathrm{mmol})$ and tetraisopropoxytitanium $(140 \mathrm{mg}, 0.52 \mathrm{mmol})$ in anhydrous $\mathrm{THF}(3 \mathrm{~mL})$ at $-78{ }^{\circ} \mathrm{C}$. The stirring was continued for $10 \mathrm{~min}$ at the same temperature. The reaction mixture was warmed to room temperature 
and heated at $50{ }^{\circ} \mathrm{C}$ for $2 \mathrm{~h}$. After cooling, the reaction was quenched with a saturated solution of $\mathrm{NH}_{4} \mathrm{Cl}(3 \mathrm{~mL})$, water $(3 \mathrm{~mL})$, and extracted with dichloromethane $(3 \times 4 \mathrm{~mL})$, the combined organic layers was washed with water, dried over anhydrous $\mathrm{MgSO}_{4}$, and filtered. The solvent was evaporated, and the residue was subjected to column chromatography on silica gel (chloroform and chloroform-ethanol 100:1 as eluent) to afford compound 2 (42 mg, 42\% yield) as a yellow powder, m.p. $91-92{ }^{\circ} \mathrm{C}$ (ether). IR $\left(\mathrm{KBr}, v, \mathrm{~cm}^{-1}\right)$ : 3047, 2974, 2935, 1736, 1701, 1628, 1585, 1474, 1390, 1356, 1286, 1226, 1195, 1120, 1034, 1011, 972, 900, 866, 840, 827, 756, 740, 700, 681. ${ }^{1} \mathrm{H}-\mathrm{NMR}\left(400 \mathrm{MHz}, \mathrm{CDCl}_{3}, \delta_{\mathrm{H}}\right): 0.88$ $\left(\mathrm{d}, J=6.9 \mathrm{~Hz}, 3 \mathrm{H}, \mathrm{CH}_{3}\right], 1.11\left(\mathrm{~d}, J=6.9 \mathrm{~Hz}, 3 \mathrm{H}, \mathrm{CH}_{3}\right), 3.20\left[\mathrm{~m}, 1 \mathrm{H}, \mathrm{CH}-\left(\mathrm{CH}_{3}\right)_{2}\right], 3.83(\mathrm{~s}, 9 \mathrm{H}$, $\left.3 \times \mathrm{OCH}_{3}\right), 6.23(\mathrm{~d}, J=9.2 \mathrm{~Hz}, 1 \mathrm{H}, \mathrm{H}-1 \mathrm{a}), 6.36(\mathrm{~d}, J=9.7 \mathrm{~Hz}, 1 \mathrm{H}, \mathrm{H}-6), 6.74(\mathrm{~d}, J=9.2 \mathrm{~Hz}, 1 \mathrm{H}, \mathrm{H}-1 \mathrm{~b})$, 6.94 (s, 1H, H-9), 7.10 (br s, 2H, H-2',6'), 7.67 (d, $J=9.7 \mathrm{~Hz}, 1 \mathrm{H}, \mathrm{H}-5), 7.90$ (s, 1H, H-4). ${ }^{13} \mathrm{C}-\mathrm{NMR}$ $\left(100 \mathrm{MHz}, \mathrm{CDCl}_{3}, \delta_{\mathrm{C}}\right): 16.1\left(\mathrm{CH}_{3}\right), 17.0\left(\mathrm{CH}_{3}\right), 29.2(\mathrm{CH}), 55.6\left(3 \times \mathrm{OCH}_{3}\right), 98.3(\mathrm{C}-2), 101.0(\mathrm{C}-9)$, 106.1 (C-2',6'), 114.8 (C-3a,4a), 115.1, 115.2 (C-6), 125.4 (C-4), 119.0 (C-1'), 126.8 (C-1a), 131.1 (C-1b), 131.8 (C-4'), 142.6 (C-5), 153.0 (C-8a), 155.3 (C-3',5'), 158.2 (C-7), 168.9 (C-9a), 192.4 (C-3). UV $(\mathrm{EtOH}) \lambda_{\max }(\lg \varepsilon): 255$ (4.44), 296 (4.07), 310 (4.01), 344 (sh), 354 (3.9) nm. Anal. Calcd for $\mathrm{C}_{25} \mathrm{H}_{24} \mathrm{O}_{7}$ : C, 68.80; H, 5.54; found C, 68.69; H, 5.17.

(Z)-2-(3-Hydroxy-4-methoxystyryl)-2-isopropyl-2H-furo[3,2-g]chromene-3,7-dione (3). Lindlar's catalyst (5 mg, $2 \mathrm{~mol} \%$ ) was added to a solution of 2-isopropyl-2-[(3-hydroxy-4-methoxy-phenyl)ethynyl]$2 \mathrm{H}$-furo[3,2-g]chromene-3,7-dione $(\mathbf{1 7}, 100 \mathrm{mg}, 0.26 \mathrm{mmol})$ in dry ethanol $(6 \mathrm{~mL})$. The system was filled with hydrogen, and the reaction mixture was stirred in a hydrogen flow for $20 \mathrm{~h}$ and then concentrated under reduced pressure. Column chromatography on silica gel afforded compound 3 (31 mg, $32 \%$ yield) as a yellow oil. IR (KBr, $\left.v, \mathrm{~cm}^{-1}\right)$ : 3520, 3488, 3398, 2960, 2925, 2852, 1750, 1726, 1724, $1630,1576,1510,1480,1463,1356,1335,1300,1250,1176,1157,1143,1105,1032,978,935,901$, $885,831,813,760,744,700,671 .{ }^{1} \mathrm{H}-\mathrm{NMR} \delta_{\mathrm{H}}\left(600 \mathrm{MHz}, \mathrm{CDCl}_{3}, \delta_{\mathrm{H}}\right): 0.87\left[\mathrm{~d}, J=6.9 \mathrm{~Hz}, 3 \mathrm{H}, \mathrm{CH}_{3}\right)$, $1.15\left[\mathrm{~d}, J=6.9 \mathrm{~Hz}, 3 \mathrm{H}, \mathrm{CH}_{3}\right), 2.37\left[\mathrm{~m}, 1 \mathrm{H}, \mathrm{C} \underline{\mathrm{H}}-\left(\mathrm{CH}_{3}\right)_{2}\right], 3.76\left(\mathrm{~s}, 3 \mathrm{H}, \mathrm{OCH}_{3}\right), 6.26(\mathrm{~d}, J=9.5 \mathrm{~Hz}, 1 \mathrm{H}$, $\mathrm{H}-1 \mathrm{~b}), 6.33$ (d, $J=9.7 \mathrm{~Hz}, 1 \mathrm{H}, \mathrm{H}-6), 6.92$ (d, $J=7.8 \mathrm{~Hz}, 1 \mathrm{H}, \mathrm{H}-5$ ') 6.79 (d, $J 9.5 \mathrm{~Hz}, 1 \mathrm{H}$, H-1b), 6.89 (dd, $J=8.0$ and $1.8 \mathrm{~Hz}, 1 \mathrm{H}, \mathrm{H}-6$ '), 7.00 (s, 1H,H-9), 7.06 (d, $J=8.0 \mathrm{~Hz}, 1 \mathrm{H}, \mathrm{H}-5$ '), 7.25 (d, $\left.J=1.8 \mathrm{~Hz}, 1 \mathrm{H}, \mathrm{H}-2^{\prime}\right), 7.68$ (d, $\left.J=9.7 \mathrm{~Hz}, 1 \mathrm{H}, \mathrm{H}-5\right), 7.78$ (s, $\left.1 \mathrm{H}, \mathrm{H}-4\right) .{ }^{13} \mathrm{C}-\mathrm{NMR}\left(150 \mathrm{MHz}, \mathrm{CDCl}_{3}, \delta_{\mathrm{C}}\right)$ : $15.6\left(\mathrm{CH}_{3}\right), 18.6\left(\mathrm{CH}_{3}\right), 31.2(\mathrm{CH}), 55.3\left(\mathrm{OCH}_{3}\right), 92.9(\mathrm{C}-2), 100.9(\mathrm{C}-9), 113.6(\mathrm{C}-6), 114.4(\mathrm{C}-5)$, 114.8 (C-3a), 116.4 (C-4a), 119.3 (C-2'), 121.7 (C-6'), 124.3 (C-4), 125.2 (C-1a), 129.2 (C-1b), 134.9 (C-1'), 143.5 (C-5), 149.2 (C-3'), 150.3 (C-4'), 159.4 (C-8a), 160.1 (C-7), 173.9 (C-9a),199.4 (C-3). UV (EtOH) $\lambda_{\max }(\lg \varepsilon): 253$ (4.41), 296 (4.08), 308 (4.06), 341 (sh), 353 (4.1) nm, Anal. Calcd for $\mathrm{C}_{23} \mathrm{H}_{20} \mathrm{O}_{6}$ : C, 70.40; H, 5.14; found C, 70.78; H, 4.92.

(Z)-2-Isopropyl-2-(2,3,4-trimethoxystyryl)-2H-furo[3,2-g]chromene-3,7-dione (6). Compound 6 (40 mg) was prepared from 2-isopropyl-2-[(2,3,4-trimethoxyphenyl)ethynyl]-2H-furo[3,2-g]chromene-3,7-dione (18, $100 \mathrm{mg}, 0.23 \mathrm{mmol}$ ) using the procedure described for 2. Yield $40 \%$, m.p. $112-114{ }^{\circ} \mathrm{C}$ (ether). IR $\left(\mathrm{KBr}, v, \mathrm{~cm}^{-1}\right): 3047,2974,1736,1701,1628,1585,1473,1391,1355,1286,1220,1196,1121,1034$, 1011, 972, 900, 866, 850, 827, 800, 756, 740, 680. ${ }^{1} \mathrm{H}-\mathrm{NMR}\left(400 \mathrm{MHz}, \mathrm{CDCl}_{3}, \delta_{\mathrm{H}}\right): 0.98(\mathrm{~d}, J=6.9 \mathrm{~Hz}$, $\left.3 \mathrm{H}, \mathrm{CH}_{3}\right), 1.19\left(3 \mathrm{H}, J=6.9 \mathrm{~Hz}, \mathrm{~d}, \mathrm{CH}_{3}\right), 3.28\left[\mathrm{~m}, 1 \mathrm{H}, \mathrm{CH}-\left(\mathrm{CH}_{3}\right)_{2}\right], 3.91\left(\mathrm{~s}, 3 \mathrm{H}, \mathrm{OCH}_{3}\right), 3.92$ (s, $\left.3 \mathrm{H}, \mathrm{OCH}_{3}\right), 3.94\left(\mathrm{~s}, 3 \mathrm{H}, \mathrm{OCH}_{3}\right), 6.23$ (d, $\left.J=9.3 \mathrm{~Hz}, 1 \mathrm{H}, \mathrm{H}-1 \mathrm{a}\right), 6.45$ (d, $\left.J=9.6 \mathrm{~Hz}, 1 \mathrm{H}, \mathrm{H}-6\right), 6.67$ (d, $\left.J=8.3 \mathrm{~Hz}, 1 \mathrm{H}, \mathrm{H}-5^{\prime}\right), 6.81$ (d, $\left.J=9.3 \mathrm{~Hz}, 1 \mathrm{H}, \mathrm{H}-1 \mathrm{~b}\right), 7.18$ (d, $\left.J=8.3 \mathrm{~Hz}, 1 \mathrm{H}, \mathrm{H}-6^{\prime}\right), 7.97$ (d, $J=9.6 \mathrm{~Hz}$, 
1H, H-5), 8.19 (s, 1H, H-4). ${ }^{13} \mathrm{C}-\mathrm{NMR}\left(100 \mathrm{MHz}, \mathrm{CDCl}_{3}, \delta_{\mathrm{C}}\right): 16.5\left(\mathrm{CH}_{3}\right), 17.4\left(\mathrm{CH}_{3}\right), 31.6(\mathrm{CH}), 55.9$ $\left(\mathrm{OCH}_{3}\right), 62.1\left(\mathrm{OCH}_{3}\right), 60.7\left(\mathrm{OCH}_{3}\right), 99.5(\mathrm{C}-2), 101.4(\mathrm{C}-9), 107.2(\mathrm{C}-5), 115.2(\mathrm{C}-6), 115.5,115.6$ (C-3a, 4a), 123.9 (C-6'), 123.0 (C-1'), 125.9 (C-4), 126.9 (C-1a), 133.1 (C-1b), 141.3 (C-5), 144.2 (C-3'), 156.6 (C-2'), 158.7 (C-4'), 159.0 (C-8a), 160.9 (C-7), 170.7 (C-9a), 190.5 (C-3). UV (EtOH) $\lambda_{\max }(\lg \varepsilon)$ : 255 (4.32), 296 (3.94), 310 (3.88), 344 (sh), 354 (3.78) nm. Anal. Calcd for $\mathrm{C}_{25} \mathrm{H}_{24} \mathrm{O}_{7}$ : C, 68.80; $\mathrm{H}$, 5.54; found C, 69.09; H, 5.42.

(Z)-2-[(3,4-Dihydroxystyryl)-2-isopropyl-2H-furo[3,2-g]chromene-3,7-dione (7). Compound 7 (24 mg) was prepared by partial reduction of 2-[(3,4-dihydroxyphenyl)ethynyl]-2-isopropyl- $\mathrm{H}$ furo[3,2-g]chromene-3,7-dione (19, $100 \mathrm{mg}, 0.26 \mathrm{mmol}$ ) using the procedure described for $\mathbf{3}$ (reaction time 30 h). Yield $24 \%$, m.p. $68-71{ }^{\circ} \mathrm{C}$ (ether). IR $\left(\mathrm{KBr}, v, \mathrm{~cm}^{-1}\right)$ : $3470,2979,2922,2851,1751,1661$, 1626, 1601, 1598, 1495, 1420, 1354, 1327, 1267, 1207, 1150, 1111, 1086, 1028, 1002, 978, 935, 914, 823, 800, 752, 696. ${ }^{1} \mathrm{H}-\mathrm{NMR}\left(400 \mathrm{MHz}, \mathrm{CDCl}_{3}, \delta_{\mathrm{H}}\right): 0.85\left(\mathrm{~d}, J=6.9 \mathrm{~Hz}, 3 \mathrm{H}, \mathrm{CH}_{3}\right), 0.91[\mathrm{~d}, J=6.9 \mathrm{~Hz}$, $\left.3 \mathrm{H}, \mathrm{CH}_{3}\right), 2.99$ [m, 1H, C$\left.-\left(\mathrm{CH}_{3}\right)_{2}\right], 6.21$ (d, $\left.J=9.5 \mathrm{~Hz}, 1 \mathrm{H}, \mathrm{H}-1 \mathrm{a}\right), 6.37$ (d, $\left.J=9.7 \mathrm{~Hz}, 1 \mathrm{H}, \mathrm{H}-6\right)$, 6.37 (d, $\left.J=8.3 \mathrm{~Hz}, 1 \mathrm{H}, \mathrm{H}-5^{\prime}\right), 6.81$ (d, $\left.J=9.5 \mathrm{~Hz}, 1 \mathrm{H}, \mathrm{H}-1 \mathrm{~b}\right), 6.89$ (s, 1H, H-9), 7.36 (d, $J=1.8 \mathrm{~Hz}$, $\left.1 \mathrm{H}, \mathrm{H}-2^{\prime}\right), 7.49$ (dd, $J=8.0$ and $\left.1.8 \mathrm{~Hz}, 1 \mathrm{H}, \mathrm{H}-6^{\prime}\right), 7.65$ (d, $\left.J=8.0 \mathrm{~Hz}, 1 \mathrm{H}, \mathrm{H}-5^{\prime}\right), 7.91$ (d, $J=9.6 \mathrm{~Hz}$, $1 \mathrm{H}, \mathrm{H}-5), 8.03$ (s, 1H, H-4). ${ }^{13} \mathrm{C}-\mathrm{NMR}\left(100 \mathrm{MHz}, \mathrm{CDCl}_{3}, \delta_{\mathrm{C}}\right): 15.1\left(\mathrm{CH}_{3}\right), 15.5\left(\mathrm{CH}_{3}\right), 30.5(\mathrm{CH})$, 98.5 (C-2), 100.9 (C-9), 115.2 (C-3a), 115.5 (C-6), 116.2 (C-5'), 117.2 (C-4a), 119.7 (C-1'), 122.2 (C-2'), 123.6 (C-6'), 125.5 (C-4), 127.1 (C-1a), 132.4 (C-1b), 142.8 (C-5), 157.9 (C-3'), 158.8 (C-4'), 159.9 (C-8a), 161.4 (C-7), 171.3 (C-9a), 190.3 (C-3). UV (EtOH) $\lambda_{\max }$ (lge): 256 (4.76), 292 (4.33), 349 (4.13) nm. Anal. Calcd for $\mathrm{C}_{22} \mathrm{H}_{18} \mathrm{O}_{6}$ : C, 69.83; H, 4.79; found C, 70.10; H, 4.31.

(Z)-2-(5-Formyl-3-hydroxy-3-methoxystyryl)-2-isopropyl-2H-furo[3,2-g]chromene-3,7-dione

Compound 8 (37 mg) was prepared from 2-[(5-formyl-3-hydroxy-3-methoxyphenyl)ethynyl]-2isopropyl-2H-furo[3,2-g] chromene-3,7-dione (20, $100 \mathrm{mg}, 0.24 \mathrm{mmol})$ using the procedure described for 3. Yield 37\%, a yellow oil. IR (KBr, $\left.v, \mathrm{~cm}^{-1}\right)$ : 3435, 3063, 3042, 2960, 2925, 2853, 2808, 1739, 1726, 1714, 1629, 1600, 1576, 1510, 1500, 1464, 1440, 1394, 1355, 1334, 1300, 1250, 1230, 1176, 1157, 1144, 1105, 1032, 978, 935, 915, 893, 831, 814, 761, 744, 720, 668. ${ }^{1} \mathrm{H}-\mathrm{NMR}$ (400 MHz, $\left.\mathrm{CDCl}_{3}, \delta_{\mathrm{H}}\right): 0.97\left(\mathrm{~d}, J=6.9 \mathrm{~Hz}, 3 \mathrm{H}, \mathrm{CH}_{3}\right], 1.07\left[\mathrm{~d}, J=6.9 \mathrm{~Hz}, 3 \mathrm{H}, \mathrm{CH}_{3}\right), 3.15\left[\mathrm{~m}, 1 \mathrm{H}, \mathrm{C} \underline{\mathrm{H}}-\left(\mathrm{CH}_{3}\right)_{2}\right]$, $3.92\left(\mathrm{~s}, 3 \mathrm{H}, \mathrm{OCH}_{3}\right), 6.23(\mathrm{~d}, J=9.3 \mathrm{~Hz}, 1 \mathrm{H}, \mathrm{H}-1 \mathrm{a}), 6.37(1 \mathrm{H}, J=9.8 \mathrm{~Hz}, \mathrm{~d}, \mathrm{H}-6), 6.41$ (d, $J=1.8 \mathrm{~Hz}$, 1H, H-6'), 6.83 (d, J=9.3 Hz, 1H, H-1b), 7.05 (s, 1H, H-9), 7.69 (d, 1H, J=9.6 Hz, H-5), 7.78 (1H, $J=1.8 \mathrm{~Hz}, \mathrm{~d}, \mathrm{H}-4$ '), 7.85 (s, 1H, H-4), 8.07 (1H, $J=8.8 \mathrm{~Hz}, \mathrm{~d}, \mathrm{H}-1 \mathrm{~b}), 9.98$ (s, 1H, CHO). ${ }^{13} \mathrm{C}-\mathrm{NMR}$ $\left(100 \mathrm{MHz}, \mathrm{CDCl}_{3}, \delta_{\mathrm{C}}\right): 15.6\left(\mathrm{CH}_{3}\right), 15.9\left(\mathrm{CH}_{3}\right), 34.0(\mathrm{CH}), 55.7\left(\mathrm{OCH}_{3}\right), 99.5(\mathrm{C}-2), 100.6(\mathrm{C}-9)$, 115.6 (C-6), 116.0 (C-3a), 116.7 (C-4a), 118.7 (C-4'), 121.8 (C-6'), 119.8 (C-1'), 126.0 (C-4), 127.5 (C-1a), 132.6 (C-1b), 133.9 (C-5'), 144.8 (C-5), 156.9 (C-3'), 158.9 (C-2'), 161.9 (C-8a), 165.3 (C-7), 171.8 (C-9a), 191.9 (CHO), 194.6 (C-3). UV (EtOH) $\lambda_{\max }(\lg \varepsilon): 256$ (4.41), 291 (3.97), 349 (3.78) nm. Anal. Calcd for $\mathrm{C}_{24} \mathrm{H}_{20} \mathrm{O}_{7}$ : C, 68.57; H, 4.80; found $\mathrm{C}, 68.24 ; \mathrm{H}, 4.68$.

2-Isopropyl-3-[(2,3,4-trimethoxyphenyl)ethynyl]-7H-furo[3,2-g]chromene-7-one (25). To a solution of 2-isopropyl-3-(trifluoromethanesulfonyloxy)psoralene (23, $150 \mathrm{mg}, 0.4 \mathrm{mmol})$ and 1-ethynyl-2,3,4trimethoxybenzene (21c, $85 \mathrm{mg}, 0.44 \mathrm{mmol})$ in benzene $(5 \mathrm{~mL})$ was added $\mathrm{CuI}(1.5 \mathrm{mg}, 2 \mathrm{~mol} \%)$, $\mathrm{Pd}\left(\mathrm{PPh}_{3}\right)_{2} \mathrm{Cl}_{2}(11 \mathrm{mg}, 4 \mathrm{~mol} \%)$, and $\mathrm{Et}_{3} \mathrm{~N}(0.076 \mathrm{~mL}, 0.55 \mathrm{mmol} ; 1.4$ equiv) under argon. The reaction 
mixture was stirred at $80^{\circ} \mathrm{C}$ for $8 \mathrm{~h}$ (TLC). The mixture was cooled and $5 \mathrm{~mL}$ of water was added. The separated water layer was extracted with methylene chloride $(5 \times 4 \mathrm{~mL})$. The combined organic extracts was washed with water, dried over $\mathrm{MgSO}_{4}$, filtered, and concentrated under reduced pressure. The residue was subjected to column chromatography on silica gel. Eluting with chloroform and crystallization from diethyl ether gave $122 \mathrm{mg}$ (73\%) of compound 25. M.p. 121-122 ${ }^{\circ} \mathrm{C}$ (ether). IR $\left(\mathrm{KBr}, v, \mathrm{~cm}^{-1}\right)$ : 3051, 2979, 2927, 2854, 2472, 2118, 1732, 1622, 1603, 1580, 1514, 1470, 1386, 1363, $1321,1277,1252,1213,1194,1167,1140,1115,1096,1068,1043,980,959,935,916,870,850,820$, 770, 750, 741, 702, 677. ${ }^{1} \mathrm{H}-\mathrm{NMR}\left(400 \mathrm{MHz}, \mathrm{CDCl}_{3}, \delta_{\mathrm{H}}\right): 1.35\left(\mathrm{~d}, J=7.0 \mathrm{~Hz}, 3 \mathrm{H}, \mathrm{CH}_{3}\right), 1.37[\mathrm{~d}$, $\left.J=7.0 \mathrm{~Hz}, 3 \mathrm{H}, \mathrm{CH}_{3}\right), 3.25\left[\mathrm{~m}, 1 \mathrm{H}, \mathrm{CH}-\left(\mathrm{CH}_{3}\right)_{2}\right], 3.86\left(\mathrm{~s}, 3 \mathrm{H}, \mathrm{OCH}_{3}\right), 3.92\left(\mathrm{~s}, 3 \mathrm{H}, \mathrm{OCH}_{3}\right), 4.01(\mathrm{~s}, 3 \mathrm{H}$, $\left.\mathrm{OCH}_{3}\right), 6.40$ (d, $\left.J=9.8 \mathrm{~Hz}, 1 \mathrm{H}, \mathrm{H}-6\right), 6.72$ (d, $\left.J=8.2 \mathrm{~Hz}, 1 \mathrm{H}, \mathrm{H}-5^{\prime}\right), 6.98$ (s, 1H, H-9), 7.59 (d, J = 8.2 Hz, 1H, H-6'), 7.79 (s, 1H, H-4), 7.80 (d, 1H, $J=9.8 \mathrm{~Hz}, \mathrm{H}-5) .{ }^{13} \mathrm{C}-\mathrm{NMR}\left(100 \mathrm{MHz}, \mathrm{CDCl}_{3}, \delta_{\mathrm{C}}\right): 20.3$ $\left(\mathrm{CH}_{3}\right), 20.5\left(\mathrm{CH}_{3}\right), 25.9(\mathrm{CH}), 56.1\left(\mathrm{OCH}_{3}\right), 60.9\left(\mathrm{OCH}_{3}\right), 62.3\left(\mathrm{OCH}_{3}\right), 86.5(\mathrm{C}-1 \mathrm{a}), 94.0(\mathrm{C}-1 \mathrm{~b}), 95.5$ (C-3), 97.5 (C-9), 107.3 (C-5'), 100.5 (C-4a), 111.1 (C-6), 116.6 (C-3a), 118.9 (C-1'), 124.2 (C-6'), 126.5 (C-4), 141.6 (C-3'), 143.5 (C-5), 152.0 (C-9a), 153.1 (C-8a), 154.1, 154.7 (C-2',4'), 156.9 (C-2), 159.2 (C-7). UV (EtOH) $\lambda_{\max }(\lg \varepsilon): 249$ (3.65), 306 (3.75), 327 (3.73), 351 (sh), 355(2.85) nm. Anal. Calcd for $\mathrm{C}_{25} \mathrm{H}_{22} \mathrm{O}_{6}$ : C, 71.76; H, 5.30; found $\mathrm{C}, 71.49 ; \mathrm{H}, 5.12$.

3-[(3,4-Dihydroxyphenyl)ethynyl]-2-isopropyl-7H-furo[3,2-g]chromene-7-one (26). Compound 26 was prepared by two methods. Reaction of psoralene derivative 23 (150 mg, $0.4 \mathrm{mmol})$ with 1-ethynyl-3,4-dihydroxybenzene $(\mathbf{2 1 d}, 60 \mathrm{mg}, 0.44 \mathrm{mmol})$ by the procedure described for $\mathbf{2 5}(\operatorname{method} a)$ gave $98 \mathrm{mg}$ (68\%) of compound 26. (b) To a solution of 3-ethynyl-2-isopropylpsoralene (32, $100 \mathrm{mg}$, $0.4 \mathrm{mmol})$ in benzene $(5 \mathrm{~mL})$ was added 3,4-dihydroxybromobenzene $(33,83 \mathrm{mg}, 0.44 \mathrm{mmol}), 1.5 \mathrm{mg}$ ( $2 \mathrm{~mol} \%$ ) of CuI, $11 \mathrm{mg}$ ( $4 \mathrm{~mol} \%$ ) of $\mathrm{Pd}\left(\mathrm{PPh}_{3}\right)_{2} \mathrm{Cl}_{2}$, and $0.076 \mathrm{~mL}$ (1.4 equiv, $\left.0.55 \mathrm{mmol}\right)$ of $\mathrm{Et}_{3} \mathrm{~N}$ under argon. The mixture was stirred at $80{ }^{\circ} \mathrm{C}$ for $10 \mathrm{~h}$ (TLC). Then the solution was cooled, $3 \mathrm{~mL}$ of water was added and reaction mixture was extracted with methylene chloride $(5 \times 4 \mathrm{~mL})$. The combined extract was washed with water, dried over $\mathrm{MgSO}_{4}$, filtered, and concentrated under reduced pressure. The residue was subjected to column chromatography on silica gel. Eluting with chloroform and crystallization from diethyl ether gave $60 \mathrm{mg}(42 \%)$ of compound 26. M.p. $104-105{ }^{\circ} \mathrm{C}$. IR $(\mathrm{KBr}$, $\left.v, \mathrm{~cm}^{-1}\right): 3450,3327,3051,2980,2880,2556,2472,2096,1732,1716,1697,1620,1590,1550,1514$, $1431,1364,1278,1253,1213,1191,1160,1140,1095,1041,980,950,876,860,849,825,770,754$, 740, 685, 650. ${ }^{1} \mathrm{H}-\mathrm{NMR}\left(400 \mathrm{MHz}, \mathrm{CDCl}_{3}, \delta_{\mathrm{H}}\right): 1.38\left(\mathrm{~d}, J=7.0 \mathrm{~Hz}, 3 \mathrm{H}, \mathrm{CH}_{3}\right), 1.40(\mathrm{~d}, J=7.0 \mathrm{~Hz}, 3 \mathrm{H}$, $\mathrm{CH}_{3}$ ), 3.30 [m, 1H, Cㅍ- $\left(\mathrm{CH}_{3}\right)_{2}$ ], 4.09 (br.s, 2H, OH), 6.32 (d, J=9.7 Hz, 1H, H-6), 7.07 (s, 1H, H-9), $7.26\left(\mathrm{~d}, J=2.0 \mathrm{~Hz}, 1 \mathrm{H}, \mathrm{H}-2^{\prime}\right), 7.32\left(\mathrm{dd}, J=8.0\right.$ and $\left.2.0 \mathrm{~Hz}, 1 \mathrm{H}, \mathrm{H}-6^{\prime}\right), 7.46\left(\mathrm{~d}, J=8.0 \mathrm{~Hz}, 1 \mathrm{H}, \mathrm{H}-5^{\prime}\right)$, $7.77(\mathrm{~d}, J=9.7 \mathrm{~Hz}, 1 \mathrm{H}, \mathrm{H}-5), 7.80$ (s, 1H, H-4). ${ }^{13} \mathrm{C}-\mathrm{NMR}\left(100 \mathrm{MHz}, \mathrm{CDCl}_{3}, \delta_{\mathrm{C}}\right): 20.0\left(\mathrm{CH}_{3}\right), 20.1$ $\left(\mathrm{CH}_{3}\right), 25.8(\mathrm{CH}), 86.4$ (C-1a), $94.0(\mathrm{C}-1 \mathrm{~b}), 100.3$ (C-3), 102.8 (C-9), 112.3 (C-4a), 115.2 (C-6), 115.3 (C-3a), 116.5 (C-1'), 120.2 (C-5'), 121.2 (C-4), 124.5 (C-2'), 126.2 (C-6'), 144.2 (C-5), 145.9 (C-3'), 147.4 (C-4'), 152.1 (C-9a), 155.3 (C-8a), 160.4 (C-2), 161.3 (C-7). UV (EtOH) $\lambda_{\max }$ (lge): 252 (3.64), 309 (3.94), 339 (3.43), 355 (sh) nm. Anal. Calcd for $\mathrm{C}_{22} \mathrm{H}_{16} \mathrm{O}_{5}$ : C, 73.33; H, 4.48; found C, 73.30; H, 4.55.

3-[(5-Formyl-3-hydroxy-3-methoxyphenyl)ethynyl]-2-isopropyl-7H-furo[3,2-g] chromene-7-one (27). Compound 27 (112 mg) was prepared from psoralene derivative 23 (150 mg, $0.4 \mathrm{mmol}$ ) and 1-ethynyl-5-formyl-2-hydroxy-3-methoxybenzene (21e, $75 \mathrm{mg}, 0.44 \mathrm{mmol}$ ) using the procedure 
described for 25. Yield 70\%, m.p. $110-112{ }^{\circ} \mathrm{C}$ (ether). IR (KBr, v, $\mathrm{cm}^{-1}$ ): 3430, 3325, 2989, 2880, 2713, 2472, 2046, 1944, 1782, 1732, 1716, 1697, 1620, 1602, 1514, 1469, 1431, 1363, 1315, 1278, 1253, 1220, 1191, 1139, 1095, 1041, 1020, 980, 904, 860, 848, 769, 754, 695, 650, 600. ${ }^{1} \mathrm{H}-\mathrm{NMR}$ $\left(400 \mathrm{MHz}, \mathrm{CDCl}_{3}, \delta_{\mathrm{H}}\right): 1.39$ (d, $\left.J=7.0 \mathrm{~Hz}, 3 \mathrm{H}, \mathrm{CH}_{3}\right), 1.42\left(\mathrm{~d}, J=7.0 \mathrm{~Hz}, 3 \mathrm{H}, \mathrm{CH}_{3}\right), 3.29$ [m, $1 \mathrm{H}$, C$\left.-\left(\mathrm{CH}_{3}\right)_{2}\right], 3.98$ (s, 3H, OCH$)_{3}, 6.36$ (d, J=9.8 Hz, 1H, H-6), 7.09 (s, 1H, H-9), 7.44 (br.s, 1H, H-4'), 7.50 (br.s, 1H, OH), 7.60 (br.s, 1H, H-6'), 7.75 (d, $J=9.8$ Hz, 1H, H-5), 7.82 (s, 1H, H-4), 10.01 (s, $1 \mathrm{H}, \mathrm{CHO}) .{ }^{13} \mathrm{C}-\mathrm{NMR}\left(100 \mathrm{MHz}, \mathrm{CDCl}_{3}, \delta_{\mathrm{C}}\right): 20.5\left(\mathrm{CH}_{3}\right), 20.6\left(\mathrm{CH}_{3}\right), 26.2(\mathrm{CH}), 60.6\left(\mathrm{OCH}_{3}\right)$, 85.6 (C-1a), 91.0 (C-1b), 99.5 (C-3), 104.4 (C-9), 115.8 (C-3a), 116.3 (C-6), 116.9 (C-4'), 118.9 (C-4a), 119.8 (C-1'), 120.6 (C-6'), 128.7 (C-4), 132.2 (C-5'), 143.9 (C-5), 150.7 (C-3'), 152.5 (C-8a), 153.1 (C-9a), 154.6 (C-2'), 159.2 (C-2), 160.7 (C-7), 191.8 (CHO). UV (EtOH) $\lambda_{\max }$ (lge): 246 (3.92), 305 (sh), 329 (3.63), 355 (2.81) nm. Anal. Calcd for $\mathrm{C}_{24} \mathrm{H}_{18} \mathrm{O}_{6}$ : C, 71.64; H, 4.51; found C, 71.30; H, 4.58.

3-[(3,4-Dihydroxyphenyl)ethynyl]-2-isopropyl-9-[(4-methylpiperazin-1-yl)methyl]-7H-furo[3,2-g]chromene-7-one (28). Compound 28 (79 mg) was prepared from 2-isopropyl-9-((4-methylpiperazin-1yl)methyl)-7-oxo-7 $H$-furo[3,2-g]chromen-3-yl trifluoromethanesulfonate (24, $195 \mathrm{mg}, 0.4 \mathrm{mmol})$ and 1-ethynyl-3,4-dihydroxybenzene (21d, $60 \mathrm{mg}, 0.44 \mathrm{mmol})$ using the procedure described for $\mathbf{2 5}$. Yield $42 \%$, yellow powder, m.p. $118-120^{\circ} \mathrm{C}$ (ether). IR (KBr, $\left.v, \mathrm{~cm}^{-1}\right): 3437,2967,2922,2851,2111,1732$, $1635,1628,1585,1511,1496,1465,1431,1388,1348,1319,1250,1213,1198,1140,1114,1095$, 1047, 957, 870, 820, 737, 700, 626, 602. ${ }^{1} \mathrm{H}-\mathrm{NMR}\left(400 \mathrm{MHz}, \mathrm{CDCl}_{3}, \delta_{\mathrm{H}}\right): 1.33$ (d, J=7.0 Hz, 3H, $\mathrm{CH}_{3}$ ), $1.36\left(3 \mathrm{H}, \mathrm{d}, J=7.0 \mathrm{~Hz}, \mathrm{CH}_{3}\right), 2.32$ (s, 3H, NCH 3 ), 2.38 (m, 4H, H-3",5"), 2.68 (m, 4H, H-2",6"), $3.25\left[\mathrm{~m}, 1 \mathrm{H}, \mathrm{C} \underline{\mathrm{H}}-\left(\mathrm{CH}_{3}\right)_{2}\right], 4.46\left(\mathrm{~d}, 1 \mathrm{H}, J=9.8 \mathrm{~Hz}, 1 \mathrm{H}, \mathrm{CH}_{2}\right), 4.58\left(\mathrm{~d}, 1 \mathrm{H}, J=9.8 \mathrm{~Hz}, 1 \mathrm{H}, \mathrm{CH}_{2}\right), 6.27$ (d, $J=9.8 \mathrm{~Hz}, 1 \mathrm{H}, \mathrm{H}-6), 6.52$ (dd, $J=8.0$ and $\left.2.0 \mathrm{~Hz}, 1 \mathrm{H}, \mathrm{H}-66^{\prime}\right), 6.64$ (d, $\left.J=2.0 \mathrm{~Hz}, 1 \mathrm{H}, \mathrm{H}-2^{\prime}\right), 6.79$ (d, $\left.J=8.0 \mathrm{~Hz}, 1 \mathrm{H}, \mathrm{H}-5^{\prime}\right), 7.59$ (d, $\left.J=9.8 \mathrm{~Hz}, 1 \mathrm{H}, \mathrm{H}-5\right), 7.74$ (s, 1H, H-4), 8.00 (br.s, 2H, OH). ${ }^{13} \mathrm{C}-\mathrm{NMR}\left(100 \mathrm{MHz}, \mathrm{CDCl}_{3}, \delta_{\mathrm{C}}\right): 20.1\left(\mathrm{CH}_{3}\right), 20.3\left(\mathrm{CH}_{3}\right), 25.9(\mathrm{CH}), 42.7\left(\mathrm{NCH}_{3}\right), 48.5\left(\mathrm{CH}_{2} \mathrm{~N}\right), 51.6$ (C-2",6"), 53.4 (C-3",5"), 85.3 (C-1a), 90.7 (C-1b), 99.2 (C-3), 104.4 (C-9), 115.0 (C-1'), 115.6 (C-3a), 116.0 (C-6), 116.6 (C-4a), 117.6 (C-5'), 122.0 (C-2'), 128.4 (C-4), 130.8 (C-6'), 143.5 (C-5), 147.1 (C-2'), 148.7 (C-4'), 152.2 (C-8a), 153.0 (C-9a), 156.9 (C-2), 160.4 (C-7). UV (EtOH) $\lambda_{\max }(\lg \varepsilon): 250$ (3.76), 284 (2.65), 327 (3.20), 346 (2.78) nm. Anal. Calcd for $\mathrm{C}_{28} \mathrm{H}_{28} \mathrm{~N}_{2} \mathrm{O}_{5}$ : C, 71.17; H, 5.97; N, 5.93; found $\mathrm{C}, 70.91 ; \mathrm{H}, 6.02 ; \mathrm{N}, 5.63$.

3-[(5-Formyl-3-hydroxy-3-methoxyphenyl)ethynyl]-2-isopropyl-9-[(4-methylpiperazin-1-yl)methyl]7H-furo[3,2-g]chromen-7-one (29). Compound 29 (119 mg) was prepared from 2-isopropyl-9-((4methylpiperazin-1-yl)methyl)-7-oxo-7H-furo[3,2-g]chromen-3-yl trifluoromethanesulfonate (24, $195 \mathrm{mg}$, $0.4 \mathrm{mmol}$ ) and 1-ethynyl-5-formyl-2-hydroxy-3-methoxybenzene (21e, $5 \mathrm{mg}, 0.44 \mathrm{mmol})$ using the procedure described for 25 . Yield 58\%, m.p. $104-105^{\circ} \mathrm{C}$ (ether). IR (KBr, $v, \mathrm{~cm}^{-1}$ ): 3402, 3117, 3080, 2958, 2850, 2783, 2711, 2611, 2172, 2133, 1726, 1693, 1610, 1593, 1537, 1464, 1427, 1402, 1367, $1331,1304,1252,1229,1213,1180,1142,1107,1065,1030,993,968,908,876,845,752,741,715$, 667, 633, 621. ${ }^{1} \mathrm{H}-\mathrm{NMR}\left(400 \mathrm{MHz}, \mathrm{CDCl}_{3}, \delta_{\mathrm{H}}\right): 1.36\left(\mathrm{~d}, J=7.0 \mathrm{~Hz}, 3 \mathrm{H}, \mathrm{CH}_{3}\right), 1.39$ (d, J=7.0 Hz, 3H, $\mathrm{CH}_{3}$ ), 2.38 (s, 3H, NCH 3 ), 2.42 (m, 4H, H-3",5"), 2.65 (m, 4H, H-2",6"), 3.25 [m, 1H, CH- $\left.\left(\mathrm{CH}_{3}\right)_{2}\right]$, $4.03\left(\mathrm{~s}, 3 \mathrm{H}, \mathrm{OCH}_{3}\right), 4.41\left(\mathrm{~d}, J=9.8 \mathrm{~Hz}, 1 \mathrm{H}, \mathrm{CH}_{2}\right), 4.48\left(\mathrm{~d}, J=9.8 \mathrm{~Hz}, 1 \mathrm{H}, \mathrm{CH}_{2}\right), 6.41(\mathrm{~d}, J=9.8 \mathrm{~Hz}$, 1H, H-6), 6.89 (br.s, 1H, H-4'), 7.41 (br.s, 1H, OH), 7.44 (br.s, 1H, H-6'), 7.57 (s, 1H, H-4), 7.79 (d, $J=9.8 \mathrm{~Hz}, 1 \mathrm{H}, \mathrm{H}-5), 9.99$ (s, 1H, CHO). ${ }^{13} \mathrm{C}-\mathrm{NMR}\left(100 \mathrm{MHz}, \mathrm{CDCl}_{3}, \delta_{\mathrm{C}}\right): 20.2\left(\mathrm{CH}_{3}\right), 20.5\left(\mathrm{CH}_{3}\right)$, 
$25.9(\mathrm{CH}), 42.5\left(\mathrm{NCH}_{3}\right), 48.2\left(\mathrm{CH}_{2} \mathrm{~N}\right), 51.5\left(\mathrm{C}-2^{\prime \prime}, 6 "\right), 52.9(\mathrm{C}-3 ", 5 "), 58.3\left(\mathrm{OCH}_{3}\right), 85.2(\mathrm{C}-1 \mathrm{a}), 93.5$ (C-1b), 100.5 (C-9), 104.2 (C-3), 115.5 (C-6), 115.7 (C-3a), 116.6 (C-4'), 118.9 (C-4a), 119.8 (C-1'), 120.5 (C-6'), 128.4 (C-4), 132.1 (C-5'), 143.5 (C-5), 150.6 (C-8a), 152.2 (C-9a), 153.4 (C-3'), 154.7 (C-2'), 156.9 (C-2), 160.4 (C-7), 189.3 (CHO). UV (EtOH) $\lambda_{\max }(\lg \varepsilon): 253$ (3.81), 289 (2.52), 328 (3.11), 350 (3.08) nm. Anal. Calcd for $\mathrm{C}_{30} \mathrm{H}_{30} \mathrm{~N}_{2} \mathrm{O}_{6}$ : C, 70.02; H, 5.88; N, 5.44; found C, 69.79; H, $5.85 ; \mathrm{N}, 5.16$.

(Z)-2-Isopropyl-3-(2,3,4-trimethoxystyryl)-2H-furo[3,2-g]chromene-3,7-dione (9). Compound 9 (60 mg) was prepared from 2-isopropyl-3-[(2,3,4-trimethoxyphenyl)ethynyl]-7H-furo[3,2-g]chromene-7-one $(\mathbf{2 5}, 100 \mathrm{mg}, 0.24 \mathrm{mmol})$ using the procedure described for 2 . Yield $60 \%$, m.p. $94-97{ }^{\circ} \mathrm{C}$ (ether). IR $\left(\mathrm{KBr}, v, \mathrm{~cm}^{-1}\right)$ : 3062, 3049, 2980, 2950, 1732, 1700, 1636, 1589, 1495, 1465, 1433, 1389, 1346, 1288, 1259, 1203, 1169, 1140, 1094, 1047, 1029, 1009, 962, 937, 903, 870, 831, 820, 781, 754, 700, 678, 650, 623, 602. ${ }^{1} \mathrm{H}-\mathrm{NMR}\left(600 \mathrm{MHz}, \mathrm{CDCl}_{3}, \delta_{\mathrm{H}}\right): 1.26\left(\mathrm{~d}, J 6.9 \mathrm{~Hz}, 3 \mathrm{H}, \mathrm{CH}_{3}\right), 1.28[\mathrm{~d}, J=6.9 \mathrm{~Hz}, 3 \mathrm{H}$, $\left.\mathrm{CH}_{3}\right), 3.16\left[\mathrm{~m}, 1 \mathrm{H}, \mathrm{C} \underline{\mathrm{H}}-\left(\mathrm{CH}_{3}\right)_{2}\right], 3.87\left(\mathrm{~s}, 3 \mathrm{H}, \mathrm{OCH}_{3}\right), 3.88\left(\mathrm{~s}, 3 \mathrm{H}, \mathrm{OCH}_{3}\right), 3.90\left(\mathrm{~s}, 3 \mathrm{H}, \mathrm{OCH}_{3}\right), 6.23(\mathrm{~d}$, $J=9.8 \mathrm{~Hz}, 1 \mathrm{H}, \mathrm{H}-6), 6.40$ (d, $J=8.8 \mathrm{~Hz}, 1 \mathrm{H}, \mathrm{H}-1 \mathrm{a}), 6.71(\mathrm{~d}, J=8.8 \mathrm{~Hz}, 1 \mathrm{H}, \mathrm{H}-1 \mathrm{~b}), 6.83$ (d, 1H, $\left.J=8.2 \mathrm{~Hz}, \mathrm{H}-5^{\prime}\right), 6.90$ (s, 1H, H-9), 7.19 (d, $\left.J=8.2 \mathrm{~Hz}, 1 \mathrm{H}, \mathrm{H}-6^{\prime}\right), 7.77$ (d, $\left.J=9.8 \mathrm{~Hz}, 1 \mathrm{H}, \mathrm{H}-5\right), 7.94$ $(\mathrm{s}, 1 \mathrm{H}, \mathrm{H}-4) .{ }^{13} \mathrm{C}-\mathrm{NMR}\left(150 \mathrm{MHz}, \mathrm{CDCl}_{3}, \delta_{\mathrm{C}}\right): 20.1\left(\mathrm{CH}_{3}\right), 20.3\left(\mathrm{CH}_{3}\right), 25.9(\mathrm{CH}), 55.3\left(\mathrm{OCH}_{3}\right)$, $\left.58.7\left(\mathrm{OCH}_{3}\right), 60.6\left(\mathrm{OCH}_{3}\right), 95.9(\mathrm{C}-3), 98.4(\mathrm{C}-9), 108.5(\mathrm{C}-3 \mathrm{a}), 114.0(\mathrm{C}-6), 115.5,115.6(\mathrm{C}-5), 4 \mathrm{a}\right)$, 123.0 (C-1'), 124.2 (C-6'), 125.9 (C-4), 130.0 (C-1a), 133.4 (C-1b), 141.5 (C-3'), 143.5 (C-5), 152.2 (C-8a), 153.0 (C-2'), 153.8 (C-4'), 154.7 (C-9a), 157.5 (C-2), 160.4 (C-7). UV (EtOH) $\lambda_{\max }(\lg \varepsilon)$ : 240 (4.25), 252 (4.14), 296 (3.82), 352 (3.49) nm. Anal. Calcd for $\mathrm{C}_{25} \mathrm{H}_{24} \mathrm{O}_{6}$ : C, 71.41; H, 5.75; found C, 71.09; H, 5.68.

(Z)-3-[(3,4-Dihydroxystyryl)-2-isopropyl-2H-furo[3,2-g]chromene-3,7-dione (10). Compound 10 (42 $\mathrm{mg})$ was prepared from 3-[(3,4-dihydroxyphenyl)ethynyl]-2-isopropyl-7 $\mathrm{H}$-furo[3,2-g]chromene-7-one (26, $100 \mathrm{mg}, 0.27 \mathrm{mmol}$ ) using the procedure described for 3. Yield $42 \%$, a yellow oil. IR (KBr, $v$, $\mathrm{cm}^{-1}$ ): 3450, 3050, 2980, 1732, 1717, 1670, 1610, 1603, 1514, 1470, 1431, 1412, 1364, 1279, 1254, 1192, 1140, 1110, 1086, 1041, 990, 975, 928, 910, 860, 849, 820, 770, 754, 740, 648, 627, 601. ${ }^{1} \mathrm{H}-\mathrm{NMR}\left(400 \mathrm{MHz}, \mathrm{CDCl}_{3}, \delta_{\mathrm{H}}\right): 1.36\left(\mathrm{~d}, J=6.9 \mathrm{~Hz}, 3 \mathrm{H},\left(\mathrm{CH}_{3}\right), 1.37\left(\mathrm{~d}, J=6.9 \mathrm{~Hz}, 3 \mathrm{H},\left(\mathrm{CH}_{3}\right), 3.25\right.\right.$ [m, 1H, C$\left.-\left(\mathrm{CH}_{3}\right)_{2}\right], 3.78$ (br.s, 2H, OH), 6.30 (d, $\left.J=9.8 \mathrm{~Hz}, 1 \mathrm{H}, \mathrm{H}-6\right), 6.39$ (d, J=9.0 Hz, 1H, H-1a), 6.65 (d, $J=9.0 \mathrm{~Hz}, 1 \mathrm{H}, \mathrm{H}-1 \mathrm{~b}), 6.80$ (d, $J=8.0 \mathrm{~Hz}, 1 \mathrm{H}, \mathrm{H}-5$ '), 6.89 (s, 1H, H-9), 7.36 (d, $J=1.6 \mathrm{~Hz}$, 1H, H-2'), 7.42 (dd, $J=8.0$ and $1.6 \mathrm{~Hz}, 1 \mathrm{H}, \mathrm{H}-6$ '), 7.79 (d, $J=9.8 \mathrm{~Hz}, 1 \mathrm{H}, \mathrm{H}-5), 7.80$ (s, 1H, H-4). ${ }^{13} \mathrm{C}-\mathrm{NMR}\left(100 \mathrm{MHz}, \mathrm{CDCl}_{3}, \delta_{\mathrm{C}}\right): 20.1\left(\mathrm{CH}_{3}\right), 20.3\left(\mathrm{CH}_{3}\right), 24.4(\mathrm{CH}), 96.0(\mathrm{C}-3), 98.4(\mathrm{C}-9)$, 106.6 (C-3a), 114.0 (C-6), 114.9, 115.1 (C-1',5'), 115.5 (C-2'), 117.2 (C-4a), 117.6 (C-6'), 125.6 (C-4), 130.9 (C-1a), 133.8 (C-1b), 143.5 (C-5), 146.5 (C-3'), 147.9 (C-4'), 152.2 (C-8a), 153.0 (C-9a), 156.4 (C-2), 160.3 (C-7). UV (EtOH) $\lambda_{\max }(\lg \varepsilon): 252$ (4.47), 287 (4.02), 350 (3.62) nm. Anal. Calcd for $\mathrm{C}_{22} \mathrm{H}_{18} \mathrm{O}_{5}$ : C, 72.92; H, 5.01; found C, 72.78; H, 4.88 .

\section{(Z)-3-(5-Formyl-3-hydroxy-3-methoxystyryl)-3-isopropyl-2H-furo[3,2-g]chromene-3,7-dione}

Compound 11 (61 mg) was prepared from 3-[(5-formyl-3-hydroxy-3-methoxyphenyl)ethynyl]-2isopropyl-7 $\mathrm{H}$-furo[3,2-g]chromene-7-one $(27,100 \mathrm{mg}, 0.24 \mathrm{mmol})$ using the procedure described for 3. Yield $60 \%$, m.p. $104-105{ }^{\circ} \mathrm{C}$ (ether). IR $\left(\mathrm{KBr}, v, \mathrm{~cm}^{-1}\right): 3435,3063,3042,2960,2925,2853,2808$, 
$1739,1726,1714,1629,1600,1576,1510,1500,1464,1440,1394,1355,1334,1300,1250,1230$, 1176, 1157, 1144, 1105, 1032, 978, 935, 915, 893, 831, 814, 761, 744, 720, 668. ${ }^{1} \mathrm{H}-\mathrm{NMR}(400 \mathrm{MHz}$, $\left.\mathrm{CDCl}_{3}, \delta_{\mathrm{H}}\right): 1.43\left(\mathrm{~d}, J=6.9 \mathrm{~Hz}, 3 \mathrm{H}, \mathrm{CH}_{3}\right), 1.46\left(\mathrm{~d}, J=6.9 \mathrm{~Hz}, 3 \mathrm{H}, \mathrm{CH}_{3}\right), 3.34$ [m, $\left.1 \mathrm{H}, \mathrm{C} \underline{\mathrm{H}}-\left(\mathrm{CH}_{3}\right)_{2}\right]$, $3.98\left(\mathrm{~s}, 3 \mathrm{H}, \mathrm{OCH}_{3}\right), 6.39$ (d, $\left.J=9.8 \mathrm{~Hz}, 1 \mathrm{H}, \mathrm{H}-6\right), 6.41$ (d, $J=1.8 \mathrm{~Hz}, 1 \mathrm{H}, \mathrm{H}-6$ '), 6.48 (d, $J=8.8 \mathrm{~Hz}$, 1H, H-1a), 6.82 (d, J=8.8 Hz, 1H, H-1b), 7.05 (s, 1H, H-9), 7.10 (br.s, 1H, OH), 7.59 (d, J=1.8 Hz, 1H, H-4'), 7.64 (s, 1H, H-4), 7.86 (d, J=9.8 Hz, 1H, H-5), 10.03 (1H, s, CHO). ${ }^{13} \mathrm{C}-\mathrm{NMR}(100 \mathrm{MHz}$, $\left.\mathrm{CDCl}_{3}, \delta\right): 20.1\left(\mathrm{CH}_{3}\right), 20.3\left(\mathrm{CH}_{3}\right), 25.8(\mathrm{CH}), 55.9\left(\mathrm{OCH}_{3}\right), 99.1(\mathrm{C}-3), 104.1(\mathrm{C}-9), 115.4(\mathrm{C}-3 \mathrm{a})$, 115.9 (C-6), 116.5 (C-4'), 117.1 (C-4a), 118.6 (C-1'), 121.4 (C-6'), 126.8 (C-4), 128.3 (C-1b), 132.1 (C-1a), 132.6 (C-5'), 143.4 (C-5), 149.9 (C-3'), 152.1 (C-8a), 152.9 (C-9a), 154.4 (C-2'), 156.8 (C-2), 160.3 (C-7), 188.2 (CHO). UV (EtOH) $\lambda_{\max }(\lg \varepsilon): 252$ (4.26), 284 (3.65), 346 (3.53) nm. Anal. Calcd for $\mathrm{C}_{24} \mathrm{H}_{20} \mathrm{O}_{6}$ : C, 70.92; H, 4.88; found $\mathrm{C}, 71.28 ; \mathrm{H}, 4.98$.

(Z)-3-[(3,4-Dihydroxystyryl)-2-isopropyl-9-[(4-methylpiperazin-1-yl)methyl]-2H-furo[3,2-g]-chromene-3,7-dione (12). The compound 12 (49 mg) was prepared from 3-[(3,4-dihydroxyphenyl)ethynyl]2-isopropyl-9-[(4-methylpiperazin-1-yl)methyl]-7H-furo[3,2-g]-chromene-7-one (28, $123 \mathrm{mg}, 0.26 \mathrm{mmol})$ using the procedure described for 3. Yield 40\%, a yellow oil. IR (KBr, v, $\mathrm{cm}^{-1}$ ): 3433, 3060, 3049, 2955, 2922, 2851, 1732, 1660, 1628, 1580, 1496, 1467, 1431, 1389, 1349, 1310, 1286, 1250, 1213, 1198, 1140, 1115, 1071, 1047, 988, 957, 905, 870, 820, 790, 768, 752, 736, 725, 708, 690, 675, 650. ${ }^{1} \mathrm{H}-\mathrm{NMR}\left(600 \mathrm{MHz}, \mathrm{CDCl}_{3}, \delta_{\mathrm{H}}\right): 1.35\left(\mathrm{~d}, J=7.0 \mathrm{~Hz}, 3 \mathrm{H}, \mathrm{CH}_{3}\right), 1.37$ (d, J=7.0 Hz, 3H, $\left.\mathrm{CH}_{3}\right), 2.28$ (s, $3 \mathrm{H}, \mathrm{CH}_{3}$ ), 2.48 (m, 4H, H-3",5"), 2.89 (m, 4H, H-2",6"), 3.25 [m, 1H, Cㅍ- $\left.-\left(\mathrm{CH}_{3}\right)_{2}\right], 4.57$ (d, J = 9.8 Hz, $\left.1 \mathrm{H}, \mathrm{CH}_{2}\right), 4.62\left(\mathrm{~d}, J=9.8 \mathrm{~Hz}, 1 \mathrm{H}, \mathrm{CH}_{2}\right), 6.28(\mathrm{~d}, J=9.7 \mathrm{~Hz}, 1 \mathrm{H}, \mathrm{H}-6), 6.37$ (d, $\left.J=9.0 \mathrm{~Hz}, 1 \mathrm{H}, \mathrm{H}-1 \mathrm{a}\right)$, 6.77 (d, $\left.J=8.3 \mathrm{~Hz}, 1 \mathrm{H}, \mathrm{H}-5^{\prime}\right), 6.95$ (d, $\left.J=9.0 \mathrm{~Hz}, 1 \mathrm{H}, \mathrm{H}-1 \mathrm{~b}\right), 7.38$ (d, $\left.J=1.8 \mathrm{~Hz}, 1 \mathrm{H}, \mathrm{H}-2^{\prime}\right), 7.49$ (dd, $J=8.0$ and $\left.1.8 \mathrm{~Hz}, 1 \mathrm{H}, \mathrm{H}-6^{\prime}\right), 7.65$ (d, $\left.J=8.0 \mathrm{~Hz}, 1 \mathrm{H}, \mathrm{H}-5^{\prime}\right), 7.78$ (s, 1H, H-4), 7.91 (d, 1H, $J=9.7 \mathrm{~Hz}$, $\mathrm{H}-5), 8.17$ (br.s, $2 \mathrm{H}, \mathrm{OH}) .{ }^{13} \mathrm{C}-\mathrm{NMR}\left(100 \mathrm{MHz}, \mathrm{CDCl}_{3}, \delta_{\mathrm{C}}\right): 19.9\left(\mathrm{CH}_{3}\right), 20.2\left(\mathrm{CH}_{3}\right), 25.6(\mathrm{CH}), 42.4$ $\left(\mathrm{CH}_{3}\right), 48.2\left(\mathrm{CH}_{2}\right), 52.8\left(\mathrm{C}-3^{\prime \prime}, 5 "\right), 51.3(\mathrm{C}-2 ", 6 "), 98.9$ (C-3), $103.9(\mathrm{C}-9), 115.2$ (C-3a), 116.3 (C-6), 117.0 (C-5'), 117.4 (C-4a), 120.0 (C-1'), 126.1 (C-2'), 127.8 (C-6'), 128.1 (C-4), 130.6, 131.6 (C-1a,1b), 143.2 (C-5), 147.1 (C-3'), 148.1 (C-4'), 151.9 (C-9a), 152.7 (C-8a), 157.7 (C-2), 160.0 (C-7). UV (EtOH) $\lambda_{\max }(\lg \varepsilon): 252$ (4.42), 275(sh), 288 (3.91), 306 (3.84), 322 (sh), 354 (3.28) nm. Anal. Calcd for $\mathrm{C}_{28} \mathrm{H}_{30} \mathrm{~N}_{2} \mathrm{O}_{5}$ : C, 70.87; H, 6.37; N, 5.90; found C, 71.02; H, 6.33; N, 5.81.

(Z)-2-(5-Formyl-3-hydroxy-3-methoxystyryl)-3-isopropyl-9-[(4-methylpiperazin-1-yl)methyl]-2H-furo[3,2-g]chromene-3, 7-dione (13). Compound 13 (56 mg) was prepared from 3-[(5-formyl-3-hydroxy-3methoxyphenyl)ethynyl]-2-isopropyl-9-[(4-methylpiperazin-1-yl)methyl]-7H-furo[3,2-g]chromen-7one $(\mathbf{2 9}, 133 \mathrm{mg}, 0.26 \mathrm{mmol})$ using the procedure described for 3. Yield 42\%, m.p. $100-102{ }^{\circ} \mathrm{C}$ (ether). IR (KBr, $v, \mathrm{~cm}^{-1}$ ): 3402, 3200, 3061, 3049, 2968, 2954, 2922, 2874, 2818, 1732, 1705, 1628, $1580,1510,1497,1466,1431,1410,1389,1348,1300,1287,1250,1214,1198,1140,1115,1070$, 1047, 1020, 975, 956, 910, 870, 820, 780, 752, 737, 720, 700, 660. ${ }^{1} \mathrm{H}-\mathrm{NMR}\left(400 \mathrm{MHz}, \mathrm{CDCl}_{3}, \delta_{\mathrm{H}}\right)$ : $1.34\left(\mathrm{~d}, J=6.9 \mathrm{~Hz}, 3 \mathrm{H},\left(\mathrm{CH}_{3}\right), 1.39\left(\mathrm{~d}, J=6.9 \mathrm{~Hz}, 3 \mathrm{H},\left(\mathrm{CH}_{3}\right), 2.28\left(\mathrm{~s}, 3 \mathrm{H}, \mathrm{CH}_{3}\right), 2.41(\mathrm{~m}, 4 \mathrm{H}\right.\right.$, H-3",5"), 2.65 (m, 4H, H-2",6"), 3.24 [m, 1H, C$\left.-\left(\mathrm{CH}_{3}\right)_{2}\right], 4.03$ (s, 3H, OCH $), 4.48$ (d, J=9.8 Hz, 1H, $\left.\mathrm{CH}_{2}\right), 4.52\left(\mathrm{~d}, J=9.8 \mathrm{~Hz}, 1 \mathrm{H}, \mathrm{CH}_{2}\right), 6.30(\mathrm{~d}, J=9.8 \mathrm{~Hz}, 1 \mathrm{H}, \mathrm{H}-6), 6.38(\mathrm{~d}, J=9.1 \mathrm{~Hz}, 1 \mathrm{H}$, H-1a), 6.45 (d, $\left.J=1.8 \mathrm{~Hz}, 1 \mathrm{H}, \mathrm{H}-6^{\prime}\right), 6.95$ (d, $\left.J=9.1 \mathrm{~Hz}, 1 \mathrm{H}, \mathrm{H}-1 \mathrm{~b}\right), 7.48$ (d, $J=1.8 \mathrm{~Hz}, 1 \mathrm{H}, \mathrm{H}-4$ '), 7.68 (s, 1H, H-4), 7.77 (d, $J=9.8 \mathrm{~Hz}, 1 \mathrm{H}, \mathrm{H}-5), 9.95$ (br.s, $1 \mathrm{H}, \mathrm{CHO}) .{ }^{13} \mathrm{C}-\mathrm{NMR}\left(100 \mathrm{MHz}, \mathrm{CDCl}_{3}\right.$, 
$\left.\delta_{\mathrm{C}}\right): 19.9\left(\mathrm{CH}_{3}\right), 20.1\left(\mathrm{CH}_{3}\right), 25.8(\mathrm{CH}), 42.5\left(\mathrm{CH}_{3}\right), 48.3\left(\mathrm{CH}_{2}\right), 51.4(\mathrm{C}-2 ", 6 "), 52.8(\mathrm{C}-3 ", 5 "), 58.3$ $\left(\mathrm{OCH}_{3}\right), 102.6(\mathrm{C}-3), 104.0$ (C-9), 115.4 (C-3a), 115.8 (C-6), 116.4 (C-4a), 118.8 (C-4'), 119.9 (C-1'), 120.0 (C-6'),125.2 (C-4), 128.6 (C-1a),131.9 (C-1b), 133.1 (C-5'), 145.1 (C-5), 145.6 (C-3'), 151.3 (C-8a), 152.0 (C-9a), 154.6 (C-2'), 156.8 (C-2), 158.9 (C-7), 189.2 (ㅌHO). UV (EtOH) $\lambda_{\max }(\lg \varepsilon): 252$ (4.59), 290 (3.82), 336 (sh), 353(3.42) nm. Anal. Calcd for $\mathrm{C}_{30} \mathrm{H}_{32} \mathrm{~N}_{2} \mathrm{O}_{6}$ : C, 69.75; H, 6.24; N, 5.42; found $\mathrm{C}, 69.52 ; \mathrm{H}, 6.38 ; \mathrm{N}, 5.35$.

2-Isopropyl-3-[(trimethylsilyl)ethynyl]-7H-furo[3,2-g]chromen-7-one (31). To a solution of oreoselone triflate 23 (200 mg, $0.5 \mathrm{mmol})$ and (trimethylsilyl)acetylene (30,73 $\mathrm{mg}, 0.75 \mathrm{mmol})$ in benzene $(5 \mathrm{~mL})$ was added $\mathrm{CuI}(1.5 \mathrm{mg}, 2 \mathrm{~mol} \%), \mathrm{Pd}\left(\mathrm{PPh}_{3}\right)_{2} \mathrm{Cl}_{2}(11 \mathrm{mg}, 4 \mathrm{~mol} \%)$, and $\mathrm{Et}_{3} \mathrm{~N}(0.076 \mathrm{~mL}, 0.55 \mathrm{mmol}$; 1.1 equiv) under argon. The reaction mixture was stirred at $80^{\circ} \mathrm{C}$ for $10 \mathrm{~h}$ (TLC). The mixture was cooled, and $5 \mathrm{~mL}$ of water was added. The separated water layer was extracted with methylene chloride $(5 \times 4 \mathrm{~mL})$. The combined organic extracts was washed with water, dried over $\mathrm{MgSO}_{4}$, filtered, and concentrated under reduced pressure. The residue was subjected to column chromatography on silica gel. Eluting with chloroform and crystallization from diethyl ether gave $90 \mathrm{mg}(58 \%)$ of compound 31, m.p. $94-96{ }^{\circ} \mathrm{C}$. IR (KBr, v, $\left.\mathrm{cm}^{-1}\right)$ : 3435, 2962, 2925, 2152, 1730, 1625, 1597, 1577, 1485, 1431, 1388, 1355, 1284, 1249, 1211, 1197, 1139, 1101, 1068, 1047, 914, 869, 754, 688. ${ }^{1} \mathrm{H}-\mathrm{NMR}\left(600 \mathrm{MHz}, \mathrm{CDCl}_{3}, \delta_{\mathrm{H}}\right): 0.16\left[9 \mathrm{H}, \mathrm{s},\left(\mathrm{CH}_{3}\right)_{3} \mathrm{Si}\right], 1.35\left(\mathrm{~d}, J=7 \mathrm{~Hz}, 3 \mathrm{H}, \mathrm{CH}_{3}\right), 1.38(\mathrm{~d}$, $\left.J=7 \mathrm{~Hz}, 3 \mathrm{H}, \mathrm{CH}_{3}\right), 3.25$ [1H, m, $\left.\mathrm{CH}\left(\mathrm{CH}_{3}\right)_{2}\right], 6.40$ (d, J=9.6 Hz, 1H, H-6), 7.40 (s, 1H, H-9), 7.57 (s, $1 \mathrm{H}, \mathrm{H}-4), 7.79$ (d, $J=9.6 \mathrm{~Hz}, 1 \mathrm{H}, \mathrm{H}-5) .{ }^{13} \mathrm{C}-\mathrm{NMR}\left(150 \mathrm{MHz}, \mathrm{CDCl}_{3}, \delta_{\mathrm{C}}\right): 9.5\left(3 \times \mathrm{CH}_{3}\right), 20.1\left(\mathrm{CH}_{3}\right)$, $20.3\left(\mathrm{CH}_{3}\right), 27.3(\mathrm{CH}), 89.2(\mathrm{C}-1 \mathrm{a}), 94.8(\mathrm{C}-1 \mathrm{~b}), 96.2$ (C-3), 98.6 (C-9), 106.9 (C-3a), 114.3 (C-6), 115.7 (C-4a), 115.8 (C-4), 116.8 (C-3), 142.7 (C-5), 152.4 (C-8a), 153.2 (C-9a), 159.0 (C-2), 160.8 (C-7). UV (EtOH) $\lambda_{\max }(\lg \varepsilon): 222$ (4.36), 251 (4.07), 294 (2.74), 338 (2.64) nm. Anal. Calcd for $\mathrm{C}_{19} \mathrm{H}_{20} \mathrm{O}_{3} \mathrm{Si}$ : C, 70.34; H, 6.21; Si 8.66; found C, 69.98; H, 5.99; Si, 8.35.

3-Ethynyl-2-isopropyl-7H-furo[3,2-g]chromen-7-one (32). To a solution of compound 31 (100 mg, $0.3 \mathrm{mmol})$ in methanol $(5 \mathrm{~mL})$ were added CsF $(230 \mathrm{mg}, 1.5 \mathrm{mmol})$ and benzyltrimethylammonium chloride (28 mg, $0.15 \mathrm{mmol}$ ). The mixture was stirred at $\mathrm{rt}$ for $10 \mathrm{~h}$ in under argon (TLC). Then $10 \mathrm{~mL}$ of water was added and the mixture was extracted with methylene chloride $(5 \times 4 \mathrm{~mL})$. The combined extract was washed with water, dried over $\mathrm{MgSO}_{4}$, filtered, and concentrated under reduced pressure. The residue was subjected to column chromatography on silica gel. Eluting with chloroform and crystallization from diethyl ether gave compound 32 (50 mg, 66\%). M.p. $82-83{ }^{\circ} \mathrm{C}$ (ether). IR ( $\mathrm{KBr}, v$, $\mathrm{cm}^{-1}$ ): 3435, 3059, 2979, 2935, 2877, 2185, 1732, 1685, 1625, 1577, 1471, 1433, 1388, 1321, 1286, 1249, 1211, 1197, 1137, 1116, 1047, 869, 819, 721, 694. ${ }^{1} \mathrm{H}-\mathrm{NMR}\left(400 \mathrm{MHz}, \mathrm{CDCl}_{3}, \delta_{\mathrm{H}}\right): 1.25(\mathrm{~d}$, $\left.J=7 \mathrm{~Hz}, 3 \mathrm{H}, \mathrm{CH}_{3}\right), 1.29\left(\mathrm{~d}, 3 \mathrm{H}, J=7 \mathrm{~Hz}, \mathrm{CH}_{3}\right), 2.37(\mathrm{~s}, 1 \mathrm{H}, \equiv \mathrm{CH}), 3.19\left[\mathrm{~m}, 1 \mathrm{H}, \mathrm{C} \underline{\mathrm{H}}\left(\mathrm{CH}_{3}\right)_{2}\right], 6.32(\mathrm{~d}$, $J=9.6 \mathrm{~Hz}, 1 \mathrm{H}, \mathrm{H}-6), 7.33$ (s, 1H, H-9), 7.50 (s, 1H, H-4), 7.71 (d, $J=9.6 \mathrm{~Hz}, 1 \mathrm{H}, \mathrm{H}-5) .{ }^{13} \mathrm{C}-\mathrm{NMR}$ $\left(100 \mathrm{MHz}, \mathrm{CDCl}_{3}, \delta_{\mathrm{C}}\right): 20.2\left(\mathrm{CH}_{3}\right), 20.4\left(\mathrm{CH}_{3}\right), 25.8(\mathrm{CH}), 80.5(\mathrm{C}-1 \mathrm{a}), 87.6(\mathrm{C}-1 \mathrm{~b}), 96.1(\mathrm{C}-3)$, 100.4 (C-9), 107.3 (C-3a), 115.6 (C-6), 116.0 (C-4a), 116.4 (C-4), 116.6 (C-3), 143.5 (C-5), 152.2 (C-8a), 153.1 (C-9a), 156.9 (C-2), 160.2 (C-7). UV (EtOH) $\lambda_{\max }$ (lge): 224 (3.91), 250 (3.94), 285 (sh), 335 (3.34) nm. Anal. Calcd for $\mathrm{C}_{16} \mathrm{H}_{12} \mathrm{O}_{3}$ : C, 76.18; H, 4.79; found C, 76.31; H, 5.09. 


\subsection{Cell Culture and Cytotoxicity Assay}

The human cancer cells of the MT-4, CEM-13 (the cells of T-cellular human leucosis), and U-937 (human monocytes) were used in this study. The cells were cultured in the RPMI-1640 medium that contained $10 \%$ embryonic calf serum, L-glutamine $(2 \mathrm{mmol} / \mathrm{L})$, gentamicin $(80 \mathrm{lg} / \mathrm{mL})$, and lincomycin $(30 \mathrm{mg} / \mathrm{mL})$ in a $\mathrm{CO}_{2}$ incubator at $37{ }^{\circ} \mathrm{C}$. The tested compounds were dissolved in DMSO and added to the cellular culture at the required concentrations. Three wells were used for each concentration. The cells which were incubated without the compounds were used as a control. Cells were placed on 96 -well microliter plates and cultivated at $37{ }^{\circ} \mathrm{C} \mathrm{C}$ in $5 \% \mathrm{CO}_{2} / 95 \%$ air for $72 \mathrm{~h}$. The cell viability was assessed through an MTT [3-(4,5-dimethylthiazol-2-yl)-2,5-phenyl-2H-tetrazolium bromide] conversion assay. 1\% MTT was added to each well. Four hours later DMSO was added and mixed for $15 \mathrm{~min}$. Optical density $(D)$ of the samples was measured on a BioRad 680 spectrophotometer Microplate Reader (BioRad, Hercules, CA, USA) at the wavelength of $570 \mathrm{~nm}$. The $50 \%$ cytotoxic dose (CTD50) of each compound (i.e., the compound concentration that causes the death of $50 \%$ of cells in a culture, or decreases the optical density twice as compared to the control wells) was calculated from the data obtained. Statistical processing of the results was performed using the Microsoft Excel-2007, STATISTICA 6.0, and GraphPad Prism 5.0 programs. The results are given as an average value \pm a deviation from the average. Reliability of differences $(p)$ was estimated using the Student $\mathrm{t}$ test. The differences with $p<0.05$ were considered as reliable. The experimental results are given as the data average values obtained from three independently conducted experiments.

\section{Conclusions}

A series of original furocoumarin derivatives having 2-(Z)- or 3-(Z)-styryl substitution in their structures have been synthesized. The cytotoxic activity of the resulting compounds against several cancer lines have been determined in the conventional MTT assay. The cytotoxicity data of compounds 2-13 demonstrate that they exhibit anticancer activity in micromolar range. Structure-activity comparison provides evidence that a $2-(Z)$-styryl substitution in the furocoumarin scaffold is preferred for cytotoxicity over the subsequent 3-(Z)-styryl substitution; the (4-methylpiperazin-1-ylmethyl) substitution in the 9-position of 3-styrylfurocoumarins increases the cytotoxic activity in MT-4 and U-937 cell lines. The biological results for the furocoumarin analogs of CA-4 1, reported herein, shown that the structural modification of furocoumarins with the introduction of (Z)-styryl moiety may prove of great importance to obtain cytotoxic anti-cancer agents.

\section{Acknowledgments}

This investigation was supported in part by the Russian Federation Basic Research (projects 12-03-92200 and 11-03-00242) and the Grants Council of the president of the Russian Federation (grants NS-3986.2012.3 and NS-2625.2014.3).

\section{Author Contributions}

The contributions of the respective authors are as follows: A. Lipeeva performed synthesis, identification, and structure elucidation of the compounds, and prepared the manuscript. M.M. 
Shakirov contributed to checking and confirming the procedures of the structural identification, especially interpretation of the NMR spectra. M.A. Pokrovsky and A.G. Pokrovsky contributed to the cytotoxicity experiments. This study was performed based on the planning of E. Shults, the corresponding author.

\section{Conflicts of Interest}

The authors declare no conflict of interest.

\section{References}

1. Pettit, G.R.; Cragg, G.M.; Singh, S.B. Antineoplastic agents, 122. Constituents of Combretum caffrum. J. Nat. Prod. 1987, 50, 386-391.

2. Lin, C.M.; Ho, H.H.; Pettit, G.R.; Hamel, E. Antimitotic natural products combretastatin A-4 and combretastatin A-2: Studies on the mechanism of their inhibition of the binding of colchicine to tubulin. Biochemistry 1989, 28, 6984-6991.

3. Bilenker, J.H.; Flaherty, K.T.; Rosen, M.; Davis, L.; Gallagher, M.; Stevenson, J.P.; Sun, W.; Vaughn, D.; Giantonio, B.; Zimmer, R.; et al. Phase I trial of combretastatin A-4 phosphate with carboplatin. Clin. Cancer Res. 2005, 11, 1527-1533.

4. Li, J.; Oyen, R.; Verbruggen, A.; Ni, Y. Small Molecule Sequential Dual-Targeting Theragnostic Strategy (SMSDTTS): From Preclinical Experiments towards Possible Clinical Anticancer Applications. J. Cancer 2013, 4, 133-145.

5. Marrelli, M.; Conforti, F.; Statti, G.A.; Cachet, X.; Michel, S.; Tillequin F.; Menichini, F. Biological potential and structure-activity relationships of most recently developed vascular disrupting agents: An overview of new derivatives of natural combretastatin A-4. Curr. Med. Chem. 2011, 18, 3035-3081.

6. Hatanaka, T.; Fujita, K.; Ohsumi, K.; Nakagawa, R.; Fukuda, Y.; Nihei, Y.; Suga, Y.; Akiyama, Y.; Tsuji, T. Novel B-ring modified combretastatin analogues: Syntheses and antineoplastic activity. Bioorg. Med. Chem. Lett. 1998, 8, 3371-3374.

7. Pérez-Melero, C.; Maya, A.B.; del Rey, B.; Peláez, R.; Caballero E.; Medarde, M. A new family of quinoline and quinoxaline analogues of combretastatins. Bioorg. Med. Chem. Lett. 2004, 14, 3771-3774.

8. Pettit, G.R.; Thornhill, A.J.; Moser, B.R.; Hogan, F. Antineoplastic Agents. 552. Oxidation of Combretastatin A-1: Trapping the $o$-quinone intermediate considered the metabolic product of the corresponding phosphate prodrug. J. Nat. Prod. 2008, 71, 1561-1563.

9. Simoni, D.; Romagnoli, R.; Baruchello, R.; Rondanin, R.; Rizzi, M.; Pavani, M.G.; Alloatti, D.; Giannini, G.; Marcellini, M.; Riccioni, T.; et al. Novel combretastatin analogues endowed with antitumor activity. J. Med. Chem. 2006, 49, 3143-3152.

10. Simoni, D.; Romagnoli, R.; Baruchello, R.; Rondanin, R.; Grisolia, G.; Eleopra, M.; Rizzi, M.; Tolomeo, M.; Giannini, G.; Alloatti, D.; et al. Novel A-Ring and B-Ring modified combretastatin A-4 (CA-4) analogues endowed with interesting cytotoxic activity. J. Med. Chem. 2008, 51, 6211-6215. 
11. Nguyen, T.T.B.; Lomberget, T.; Tran, N.C.; Colomb, E.; Nachtergaele, L.; Thoret, S.; Dubois, J.; Guillaume, J.; Abdayem, R.; Haftek, M.; et al. Synthesis andbiological evaluation of novel heterocyclic derivatives of combretastatin A-4. Bioorg. Med. Chem. Lett. 2012, 22, 7227-7231.

12. Nguyen, T.T.B.; Lomberget, T.; Tran, N.C.; Barret, R. Synthesis of (Z) isomers of benzoheterocyclic derivatives of combretastatin A-4: A comparative study of several methods. Tetrahedron 2013, 69, 2336-2347.

13. Santana L.; Uriarte, E.; Roleira, F.; Milhazes, N.; Borges, F. Furocoumarins in medicinal chemistry: Synthesis, natural occurrence and biological activity. Curr. Med. Chem. 2004, 11, 3239-3261.

14. Wu, L.; Wang, X.; Xu, W.; Farzaneh F.; Xu, R. The structure and pharmacological functions of coumarins and their derivatives. Curr. Med. Chem. 2009, 16, 4236-4260.

15. Conforti, F.; Marrelli, M.; Menichini, F.; Bonesi, M.; Statti, G.; Provenzano, E.; Menichini, F. Natural and synthetic furanocoumarins as treatment for Vitiligo and Psoriasis. Curr. Drug Ther. 2009, 4, 38-58.

16. Viola, G.; Vedaldi, D.; Dall'Acqua, F.; Fortunato, E.; Basso, G.; Bianchi, N.; Zuccato, C.; Borgatti, M.; Lampronti, I.; Gambari, R. Induction of $\gamma$-globin mRNA, erythroid differentiation and apoptosis in UVA-irradiated human erythroid cells in the presence of furocoumarin derivatives. Biochem. Pharmacol. 2008, 2, 810-825.

17. Salvador, A.; Brognara, E.; Vedaldi, D.; Castagliuolo, I.; Brun, P.; Zuccato, C.; Lampronti I.; Gambari, R. Induction of erythroid differentiation and increased globin mRNA production with furocoumarins and their photoproducts. J. Photochem. Photobiol. B Biol. 2013, 121, 57-66.

18. Via, L.D.; González-Gómez, J.C.; Pérez-Montoto, L.G.; Santana, L.; Uriarte, E.; Magno, S.M.; Gia, O. A new psoralen derivative with enlarged antiproliferative properties. Bioorg. Med. Chem. Lett. 2009, 19, 2874-2876.

19. Lipeeva, A.V.; Shul'ts, E.E.; Shakirov, M.M.; Tolstikov, G.A. Plant coumarins. XI. Cross-coupling reactions of 2(tosyl)oreoselone. Russ. J. Org. Chem. 2013, 49, 99-107.

20. Osadchii, S.A.; Shul'ts, E.E.; Shakirov, M.M.; Tolstikov, G.A. Study of Plant Coumarins. 1. Transformations of peucedanin. Russ. Chem. Bull. (Int. Ed.) 2006, 55, 375-379.

21. Lawrence, N.J.; Ghani, F.A.; Hepworth, L.A.; Hadfield, J.A.; McGown, A.T.; Pritchard, R.G. The Synthesis of $(E)$ and $(Z)$-Combretastatins A-4 and a Phenanthrene from Combretum caffrum. Synthesis 1999, 1656-1660.

22. Lara-Ochoa, F.; Espinosa-Pérez, G. A new synthesis of combretastatins A-4 and AVE-8062A. Tetrahedron Lett. 2007, 48, 7007-7010.

23. Petrov, O.I.; Gerova, M.S.; Chanev, C.D.; Petrova, K.V. New Efficient Synthesis of Combretastatin A-4 via Colvin Rearrangement. Synthesis 2011, 3711-3715.

24. Lipeeva, A.V.; Shul'ts, E.E.; Bagryanskaya, I.Yu.; Shakirov, M.M.; Tolstikov, G.A. Plant coumarins. VI. Synthesis of 3-vinylfurocoumarin derivatives based on oreoselone. Russ. J. Org. Chem. 2011, 47, 1083-1090.

25. Lipeeva, A.V.; Shul'ts, E.E.; Shakirov, M.M.; Bagryanskaya, I.Yu.; Tolstikov, G.A. Plant coumarins. XIII. Synthesis of 2,3,9-trisubstituted furocoumarins. Russ. J. Org. Chem. 2013, 49, 403-411.

26. Lipeeva, A.V.; Shul'ts, E.E.; Shakirov, M.M.; Tolstikov, G.A. Synthesis of (Z)-styrylfurocoumarins as the heterocyclic analogues of combretastatins. Dokl. Chem. 2012, 447, 282-285. 
27. Wilson, J.K.; Sargent, J.M.; Elgie, A.W.; Hill, J.G.; Taylor, C.G. A feasibility study of the MTT assay for chemosensitivity testing in ovarian malignancy. Br. J. Cancer 1990, 62, 189-194.

28. Pettit, G.R.; Grealish, M.P.; Jung, M.K.; Hamel, E.; Pettit, R.K.; Chapuis, J.C.; Schmidt, J.M. Antineoplastic agents. 465. Structural modification of resveratrol: Sodium resverastatin phosphate. J. Med. Chem. 2002, 45, 2534-2542.

29. Gaukroger, K.; Hadfield, J.A.; Lawrence, N.J.; Nolan, S.; McGown, A.T. Structural requirements for the interreaction of combretastatins with tubulin: How important is the trimethoxy unit? Org. Biomol. Chem. 2003, 1, 3033-3037.

30. Álvarez, C.; Álvarez, R.; Corchete, P.; Pérez-Melero, C.; Peláez, R.; Medarde, M. Exploring the effect of 2,3,4-trimethoxy-phenyl moiety as a component of indolephenstatins. Eur. J. Med. Chem. 2010, 45, 588-597.

31. Ferguson, G.; McCrindle, R.; McAlees A.J.; Parvez, M. trans-Dichlorobis(triphenylphosphine)palladium(II). Acta Cryst. 1982, 38, 2679-2681.

32. Odlo, K.; Hentzen, J.; Fournier dit Chabert, J.; Ducki, S.; Gani, O.A.; Sylte, I.; Skrede, M.; Flörenes, V.A.; Hansen, T.V. 1,5-Disubstituted 1,2,3-triazoles as cis-restricted analogues of combretastatin A-4: Synthesis, molecular modeling and evaluation as cytotoxic agents and inhibitors of tubulin. Bioorg. Med. Chem. 2008, 16, 4829-4838.

33. Ito, Y.; Aoyama, T.; Shioiri, T. A new preparation of benzofurans utilizing trimethylsilyl-diazomethane. Synlett 1997, 1163-1164.

34. Gangjee, A.; Namjoshi, O.A.; Keller, S.N.; Smith, C.D. 2-Amino-4-methyl-5-phenylethyl substituted-7-N-benzylpyrrolo[2,3-d]pyrimidines as novel antitumor antimitotic agents that also reverse tumor resistance. Bioorg. Med. Chem. 2011, 19, 4355-4365.

35. Ghasemnejad-Bosra, H.; Ramzanian-Lehmali, F.; Jafari, S. Simple and improved regioselective brominations of aromatic compounds using $N$-benzyl- $N, N$-dimethylanilinium peroxodisulfate in the presence of potassium bromide under mild reaction conditions. J. Serb. Chem. Soc. 2011, 76, 685-692.

36. Nair, R.N.; Lee, P.J.; Grotjahn, D.B.; Rheingold, A.L. Single bifunctional ruthenium catalyst for one-pot cyclization and hydration giving functionalized indoles and benzofurans. Chem. Eur. J. 2010, 16, 7992-7995.

37. Tajik, H.; Mohammadpoor-Baltork, I.; Albadi, J. Bromination of some aromatic compounds with potassium bromide in the presence of benzyltriphenylphosphonium peroxodisulfate. Synth. Commun. 2007, 37, 323-328.

Sample Availability: Samples of the compounds reported in this paper are available from the authors.

(C) 2014 by the authors; licensee MDPI, Basel, Switzerland. This article is an open access article distributed under the terms and conditions of the Creative Commons Attribution license (http://creativecommons.org/licenses/by/3.0/). 